Pacific Journal of Mathematics

INVARIANCE OF PROPERTIES UNDER AUTOMORPHISMS OF
THE LATTICE OF RECURSIVELY ENUMERABLE SETS 


\section{INVARIANCE OF PROPERTIES UNDER AUTOMORPHISMS OF THE LATTICE OF RECURSIVELY ENUMERABLE SETS}

\section{Michael Stob}

1. Introduction. An important question about the structure of $\mathscr{E}$, the lattice of r.e. sets, is the study of which properties of r.e. sets are invariant under Aut $\mathscr{E}$, the group of automorphisms of $\mathscr{E}$. Many of the more important properties of r.e. sets are easily seen to be invariant. Simplicity, maximality, and $r$-maximality are invariant since the usual definitions are in terms of the lattice operations of $\mathscr{E}$. Hyperhypersimplicity is invariant since Lachlan has characterized the hyperhypersimple sets as those which have Boolean algebras for their lattices of r.e. supersets. D. A. Martin has shown that hypersimplicity is not invariant. We show in this paper that dense simplicity is not invariant.

Definition 1.1. (a) If $B \subseteq N, p_{B}$ is the function that enumerates the elements of $B$ in increasing order; i.e., $p_{B}(n)=n$th element of $B$.

(b) A coinfinite r.e. set $A$ is hypersimple (Post) if $p_{\bar{A}}$ is not dominated by any total recursive function (i.e., for each recursive function $f$ there are infinitely many $n$ for which $f(n)<p_{\bar{A}}(n)$ ).

(c) A coinfinite r.e. set $A$ is dense simple (Martin), if $p_{\bar{A}}$ dominates every total recursive function. (It is clear that if $A$ is dense simple, $A$ is hypersimple.)

THEOREM 1.2. There is a dense simple set $A$, an r.e. set $B$ which is not hypersimple, and an automorphism $\Phi$ of $\mathscr{E}$ such that $\Phi(A)=B$.

Corollary 1.3. Dense simplicity is not invariant under Aut $\mathscr{E}$.

CoROLlary 1.4 (D. A. Martin). Hypersimplicity is not invariant under Aut $\mathscr{E}$.

A proof of Martin's theorem as well as a summary of most of what is known about the automorphisms of $\mathscr{E}$ may be found in [5]. Martin's proof was a finite injury priority argument. In [5], however, Soare gives a powerful new technique of the infinite injury type for constructing automorphisms and uses this to prove that the maximal sets form an orbit of Aut $\mathscr{E}$. It is this technique that 
we use and extend to prove Theorem 1.2.

In $\S 2$ we give the basic strategy for meeting each of the requirements of the theorem. In $\S 3$ we describe the "pinball machines" which give the basic combinatorial picture of the proof and we give the basic construction. In $\S 4$ we give the rules describing the movement of "balls" on the "pinball machines" and thereby determining the sets $A, B$ and the automorphism $\Phi$. In $\S 5$ we carry out the verification that the construction works. In $\S 6$ we discuss some open problems suggested by this theorem.

Our notation is for the most part standard; a reference is Rogers [3]. We identify a set with its characteristic function and let $A[x]$ denote the restriction of $A$ to arguments $\leqq x . \quad N$ always denotes the set of nonnegative integers. Let $\mathscr{C}^{*}$ denote the lattice $\mathscr{E}$ modulo $\mathscr{F}$ where $\mathscr{F}$ is the ideal of finite sets. If $A, B \in \mathscr{E}$, $A^{*}, B^{*} \in \mathscr{E}^{*}$ denote the equivalence classes of $A, B$. We let $A^{*}=B^{*}$ be denoted by $A={ }^{*} B$ (i.e., the symmetric difference of $A$ and $B$ is finite); $A \cong{ }^{*} B$ denotes $A \cap \bar{B}={ }^{*} \phi$; and $B \subset_{\infty} A$ denotes $A-B$ is infinite. Often we will (implicitly) confuse $A$ and $A^{*}$ by choosing a particular representative of $A^{*}$. A recursive array is a recursive sequence of r.e. sets. A simultaneous enumeration of a given recursive array $\left\{X_{n}\right\}_{n \in N}$ is a $1: 1$ recursive function $g$ with range $\left\{\langle m, n\rangle: m \in X_{n}\right\}$. Thus at each state $s, g(s)=\langle m, n\rangle$ enumerates one element $m$ in one r.e. set $X_{n}$. If $g$ is assumed, $X_{n, s}$ denotes the set of elements enumerated in $X_{n}$ by the end of stage $s, X_{n} \backslash X_{m}=$ $\left\{x: \exists s\left(x \in X_{n, s}-X_{m, s}\right)\right\}$, and $X_{n} \backslash X_{m}=\left(X_{n} \backslash X_{m}\right) \cap X_{m}$. The e-state of an element $x$ at stage $s$ is $\sum_{i \leqq e ; x \in X_{i, s}} 2^{e-i}$ and is denoted by $\sigma(s, e, x)$. We sometimes identify $\sigma(s, e, x)$ with $\left\{i \leqq e: x \in X_{i, s}\right\}$.

2. The requirements. The strategy for making $A$ dense simple is straightforward. If $\left\{\varphi_{i}\right\}_{i \in N}$ is an effective listing of all (partial) recursive functions, we simply insure that $\varphi_{i}(j)<p_{\bar{A}}(n)$ for all $i, j \leqq n$. Note that the only negative requirment on $A$ is to make $\bar{A}$ infinite; we have considerable freedom in enumerating elements in $A$ beyond those necessary to insure $p_{\bar{A}}$ dominant.

To guarantee that $B$ is not hypersimple, we will use an alternative characterization of hypersimplicity. If $x_{1}<x_{2}<\cdots<x_{k}$ and $y=2^{x_{1}}+\cdots+2^{x_{k}}$, let $D_{y}$ denote the finite set $\left\{x_{1}, \cdots, x_{k}\right\}$. A sequence of r.e. sets $\left\{D_{h(n)}\right\}_{n \in N}$ determined by a recursive function $h$ is a strong array. Kuznecov, Medvedev, and Uspenskii (see [3]) have shown that our definition of hypersimplicity is equivalent to the nonexistence of a strong array $\left\{D_{h(n)}\right\}_{n \in N}$ of pairwise disjoint sets such that $D_{h(n)} \cap \bar{B} \neq \phi$ for all $n$. Fix a strong array $\left\{D_{h(n)}\right\}_{n \in N}$ of disjoint sets such that $\left|D_{h(n)}\right|=n+1$ and $\bigcup_{n} D_{h(n)}=N$. We 
will arrange that $\left|D_{h(n)} \cap B\right| \leqq n$. This requirement is clearly a negative requirement on $B$.

We will not actually construct $\Phi \in$ Aut $\mathscr{E}$ so that $\Phi(A)=B$. Rather, we will construct $\Phi \in$ Aut $\mathscr{E}^{*}$ such that $\Phi\left(A^{*}\right)=B^{*}$. Soare shows [5, Corollary 1.5] that this is enough. Soare also notes [5, p. 85] that all known constructions of $\Phi \in$ Aut $\mathscr{C}^{*}$ are accomplished by constructing a permutation $p$ of $N$ inducing $\Phi$ (i.e., $p(C)={ }^{*} \Phi(C)$ ) and functions $f$ and $g$ so that

$$
\Phi\left(W_{n}^{*}\right)=W_{f(r)}^{*} \text { and } \Phi^{-1}\left(W_{n}^{*}\right)=W_{y(n)}^{*} .
$$

Of course $p$ guarantees that $\Phi$ preserves inclusion and $f$ and $g$ guarantee that $\Phi$ and $\Phi^{-1}$ map $\mathscr{E}^{*}$ to $\mathscr{E}^{*}$. To meet the two different requirements of (1), we will enumerate two recursive arrays $\left\{V_{n}\right\}_{n \in N}$ and $\left\{U_{n}\right\}_{n \in N}$ such that $U_{n}={ }^{*} V_{n}={ }^{*} W_{n}$ for all $n$ and we will simultaneously enumerate recursive arrays $\left\{\hat{U}_{n}\right\}_{n \in N}$ and $\left\{\hat{V}_{n}\right\}_{n \in N}$ so that:

$$
p\left(U_{n}\right)={ }^{*} \hat{U}_{n} \text { and } p^{-1}\left(V_{n}\right)={ }^{*} \hat{V}_{n} .
$$

The requirements (2) can be naturally split into two parts corresponding to $A$ and $\bar{A}$. We will satisfy for each $n$ both

$$
p\left(\bar{A} \cap U_{n}\right)={ }^{*} \bar{B} \cap \hat{U}_{n} \text { and } p^{-1}\left(\bar{B} \cap V_{n}\right)={ }^{*}\left(\bar{A} \cap \hat{V}_{n}\right),
$$

and

$$
p\left(A \cap U_{n}\right)={ }^{*}\left(B \cap \hat{U}_{n}\right) \text { and } p^{-1}\left(B \cap V_{n}\right)={ }^{*}\left(A \cap \hat{V}_{n}\right) .
$$

Let $\hat{U}_{n}=\hat{U}_{n}^{+} \cup \hat{U}_{n}^{-}$where $\hat{U}_{n}^{+}=\hat{U}_{n} \backslash B$ and $\hat{U}_{n}^{-}=B \backslash \hat{U}_{n}$. Similarly let $\hat{V}_{n}=\hat{V}_{n}^{+} \cup \hat{V}_{n}^{-}$where $\hat{V}_{n}^{+}$and $\hat{V}_{n}^{-}$are defined with $A$ in place of $B$. Condition (3) causes us only to enumerate elements in the sets $\hat{U}_{n}^{+}$and $\hat{V}_{n}^{+}$. Condition (4) should only require us to enumerate elements in $\hat{U}_{n}^{-}$and $\hat{V}_{n}^{-}$. However, these requirements are not quite independent as $\hat{U}_{n}^{+} \searrow B \neq \phi$ and $\hat{V}_{n}^{+} \searrow A \neq \phi$. To see how this might affect meeting (4) in our case, consider the following situation. Suppose $\left|V_{n} \cap \bar{B}\right|$ is large so that we must enumerate many elements of $\bar{A}$ in $\hat{V}_{n}^{+}$. Later, to make $A$ dense simple, many of these elements must be enumerated in $A$. But then we must enumerate many elements of $V_{n} \cap \bar{B}$ into $B$ to meet (4), but these might be restrained by the requirements to make $B$ nonhypersimple. It is easy to see here that the positive requirement on $B$ is $\left|V_{n}^{+} \searrow A\right|=$ $\infty \Longrightarrow\left|V_{n} \searrow B\right|=\infty$.

In general, Soare gives necessary and sufficient conditions [5, Theorem 2.2, p. 91] on the enumeration of $A$ and $B$ so that the permutation $p$ guaranteeing (4) exists. We will state these conditions precisely in $\S 5$. We will control the enumerations of $A$ and $B$ to meet Soare's conditions. Thus, (4) will be met by [5, Theorem 
2.2]. We remark here that the requirements of Soare on the enumeration of $A$ and $B$ can be phrased as purely positive requirements. Thus they conflict with the requirements on $B$, which are negative, but only conflict with the requirements on $A$ to make $\bar{A}$ infinite.

We are thus left only with the requirements (3). Namely, we must construct a permutation $p \mid \bar{A}$ and enumerate r.e. sets $\left\{\hat{U}_{n}^{+}\right\}_{n \in N}$ and $\left\{\hat{V}_{n}^{+}\right\}_{n \in N}$ so that

$$
p\left(\bar{A} \cap U_{n}\right)={ }^{*}\left(\bar{B} \cap \hat{U}_{n}^{+}\right) \text {and } p^{-1}\left(\bar{B} \cap V_{n}\right)={ }^{*}\left(\bar{A} \cap \hat{V}_{n}^{+}\right) .
$$

Now these requirements are the same requirements that Soare had to meet in proving [5, Theorem 2.2] but for $A, B, \hat{U}_{n}, \hat{V}_{n}$ in place of $\bar{A}, \bar{B}, \hat{U}_{n}^{+}, \hat{V}_{n}^{+}$. Our construction of $p$ is therefore similar to Soare's. There is one complication increasing the difficulty of our task, however. In Soare's case, since $A$ and $B$ are r.e., no elements "leave" the domain of $p$ and $p^{-1}$. Here, however, we are constantly enumerating elements in $A$ to make $A$ dense simple and elements in both $A$ and $B$ to satisfy Soare's conditions for (4) to hold. The reader familiar with [5, Theorem 2.2] will see that this is the main new feature of our proof and that it adds considerable complexity to the construction.

The first example of an argument in which requirements (5) are met is in [6] where Soare proves that the lattice of supersets of a low r.e. set $A\left(A^{\prime} \equiv_{T} \varnothing^{\prime}\right)$ is isomorphic to $\mathscr{E}$. The main technical device for meeting such requirements introduced by him is the assignment of certain priorities so that the domain of $p$ settles down. It is this device which we use in a different way to meet the requirements. We have written this paper and [6] simultaneously, so as to develop a common framework for automorphism arguments which feature requirements of the form (5). While writting this paper we have benefitted from numerous suggestions of W. Maass.

3. The construction. We assume that we are given a recursive function $g$ which enumerates simultaneously $\left\{W_{n}\right\}_{n \in N^{\prime}}\left\{\hat{W}_{n}\right\}_{n \in N^{\prime}}$ and computations $\varphi_{i, s}(j), i, j, s \in N$, where $\left\{W_{n}\right\}_{n \in N}$ and $\left\{\hat{W}_{n}\right\}_{n \in N}$ are standard enumerations of the r.e. sets. We will also assume for convenience that $W_{0}=\hat{W}_{0}=N$ and $g$ enumerates $x \in W_{0}\left(\hat{W}_{0}\right)$ before enumerating $x \in W_{e}\left(\hat{W}_{e}\right)$ for any $e>0$. Further, we assume that, if $x \in W_{e}, x$ is enumerated in $W_{e}$ at infinitely many different stages.

We will enumerate in stages recursive arrays $\left\{U_{n}\right\}_{n \in N^{\prime}}\left\{V_{n}\right\}_{n \in N^{\prime}}$ $\left\{\hat{U}_{n}^{+}\right\}_{n \in N^{\prime}}\left\{\hat{V}_{n}^{+}\right\}_{n \in N}$ and r.e. sets $A$ and $B$ and construct a permutation $p$ so that all the requirements described in $\S 2$ are met. 
We will, like Soare, present the proof as a construction on pinball machines. The pinball machine $M$ is shown in Figure $1 ; \hat{M}$ is identical except that each symbol $X$ is replaced by $\hat{X}$. Pocket $\hat{A}$ will also be referred to as pocket $B$. Two copies of $N,\{n\}_{n \in N}$ and $\{\hat{n}\}_{n \in N}$ act as the balls of $M$. and $\hat{M}$. A ball $x(\hat{x})$ enters $M(\hat{M})$ from hole $H_{1}\left(\hat{H}_{1}\right)$. It then proceeds along the surface of the machine, that portion of the machine covered by arrows, until it reaches a pocket. From a pocket, $x(\hat{x})$ may re-enter the machine from hole $H_{2}, H_{3}, H_{4}$.

We now describe what the various parts of the machines are for. Balls entering pocket $A(\hat{A})$ represent numbers enumerated in $A(B)$. Balls elsewhere in the machine $M(\hat{M})$ are numbers in $\bar{A}(\bar{B})$. Thus no ball is allowed to leave pocket $A(\hat{A})$. When $x(\widehat{x})$ is at gate $G_{2}\left(\widehat{G}_{2}\right)$ we determine whether to enumerate $x \in A(B)$ to meet the conditions of the Extension Theorem of Soare [5, Theorem 2.2]. Hole $H_{4}$ is used to place $x \in A$ to make $A$ dense simple. The rest of the machine is used to guarantee (5). Before describing

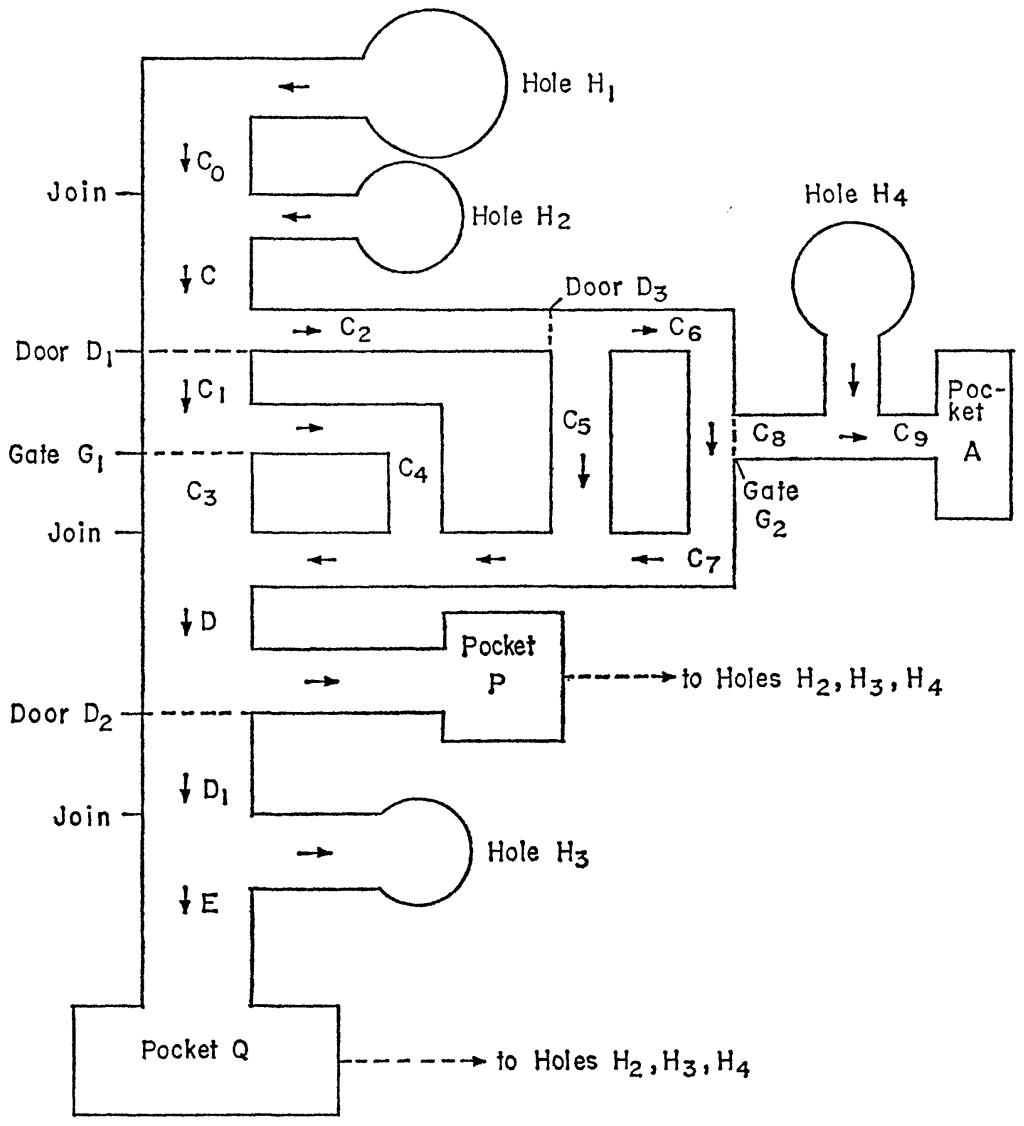

Figure 1 
how this is done we need some notation and definitions.

If $x \in M_{s}$, the full $e$-state of $x$ at stage $s$ denoted $\nu(s, e, x)$ is the triple $\langle e, \sigma(s, e, x), \tau(s, e, x)\rangle$ where $\sigma(s, e, x)$ and $\tau(s, e, x)$ denote the $e$-states of $x$ at stage $s$ measured with respect to $\left\{U_{n}\right\}_{n \in N}$ and $\left\{\hat{V}_{n}^{+}\right\}_{n \in N}$ respectively. We will use $e$-states as finite sets of integers $\leqq e$. If $\widehat{x} \in \hat{M}_{s}, \nu(s, e, \widehat{x})$ is $\langle e, \sigma(s, e, \hat{x}), \tau(s, e, \hat{x})\rangle$ where $\sigma(s, e, \widehat{x})$ and $\tau(s, e, \hat{x})$ are $e$-states measured with respect to $\left\{\hat{U}_{n}^{+}\right\}_{n \in N}$ and $\left\{V_{n}\right\}_{n \in N}$. if $\sigma, \tau$ are $e$-states and $\nu=\langle e, \sigma, \tau\rangle$ a full $e$-state, $[\sigma]_{i}=\sigma \cap\{0,1, \cdots, i\}$ and $[\nu]_{i}=\left\langle i,[\sigma]_{i},[\tau]_{i}\right\rangle$. Let $\nu_{-1}=\langle-1, \phi, \phi\rangle$. Let $\nu=\langle e, \sigma, \tau\rangle$ and $\nu^{\prime}=\left\langle e^{\prime}, \sigma^{\prime}, \tau^{\prime}\right\rangle$. We say $\nu^{\prime}$ extends $\nu$, written $\nu \precsim \nu^{\prime}$ if $e \leqq e^{\prime},\left[\sigma^{\prime}\right]_{e}=$ $\sigma$, and $\left[\tau^{\prime}\right]_{e}=\tau$. We say $\nu \leqq \nu^{\prime}$ if $e=e^{\prime}, \sigma \leqq \sigma^{\prime}$, and $\tau \supseteqq \tau^{\prime}$. Let $\nu \leqq^{\tau} \nu^{\prime}\left(\nu \leqq{ }^{\sigma} \nu^{\prime}\right)$ denote $\nu \leqq \nu^{\prime}$ and $\tau=\tau^{\prime}\left(\sigma=\sigma^{\prime}\right)$. If $\mathscr{S}$ is a set of full $e$-states, let $\mathscr{S}\left[\nu_{0}\right]=\left\{\nu: \nu \in \mathscr{S}\right.$ and $\left.\nu_{0} \precsim \nu\right\}$. If $\nu=\langle e, \sigma, \tau\rangle$, the length of $\nu,|\nu|$, is $e$.

A track is a section of the surface of the machine between any two of the following: door, gate, pocket, or join. If $X$ is a track of $M$, let $\mathscr{S}_{s}(X)=\{\nu(s, e, x): e \leqq x$ and $x$ enters track $X$ at stage $s\}$, arranged as a sequence in order of $e$. Let $\mathscr{S}(X)$ be the concatenation of the $\mathscr{S}_{s}(X), s \in N$. Such a sequence of full $e$-states is called stream $X$. If $\nu \in \mathscr{S}(X)$ infinitely often, we write $\nu \in \mathscr{S}(X)$ i.o. $\mathscr{S}(\hat{X})$ for $\hat{X}$ a track of $\hat{M}$ is defined similarly. We say a stream $X$ covers (dual covers, $\tau$-exactly covers, exactly covers) $\nu$ if some $\nu^{\prime} \in \mathscr{S}(\hat{X})$ i.o. where $\nu \leqq \nu^{\prime}\left(\nu^{\prime} \leqq \nu, \nu \leqq \tau \nu^{\prime}, \nu=\nu^{\prime}\right)$. Steam $X$ covers steam $Y$ if $X$ covers every $\nu$ such that $\nu \in \mathscr{S}(Y)$ i.o. Streams $X$ and $Y$ are equivalent if each exactly covers the other.

We now describe the role of pockets $P$ and $\hat{Q}, \hat{P}$ and $Q$ are similar. To construct a permutation satisfying (5), we would like to pair elements $x \in M$ and $\hat{y} \in \hat{M}$ which are in the same full $e$-state. Pocket $\hat{Q}$ will consist of elements $\hat{y}$ needing mates. We will attempt to choose mates for the elements of $\hat{Q}$ from those elements $x$ entering track $D$. We will choose such an $x$ as a mate for $\hat{y}$ at stage $s+1$ if $\nu(s, d, x)=\nu(s, d, \hat{y})$ where $d$ depends on $s$ and $\hat{y}$. We allow $d$ to be small when few $x$ appear in any appropriate state making it easier to choose a mate for $\hat{y}$. For most (i.e., almost all) $\hat{y}$, however, we must have $d$ be large so as to guarantee (5). Of course, to insure that $x$ 's do appear that are appropriate mates for $\hat{y}$, we must, in general, enumerate $x(\hat{y})$ in some sets $V_{n}^{+}\left(U_{n}^{+}\right)$. For instance, suppose $\nu \in \mathscr{S}(\hat{E})$ i.o. That is, infinitely many $\hat{y}$ appear on the track before pocket $\hat{Q}$ in state $\nu$. Suppose that $\nu^{\prime} \in \mathscr{S}(C)$ i.o. where $\nu^{\prime} \geqq \nu$. Let $\nu^{\prime}=\left\langle e, \sigma^{\prime}, \tau^{\prime}\right\rangle$ and $\nu=\langle e, \sigma, \tau\rangle$. Then it is possible to match up the $\hat{y}$ in state $\nu$ with the $x$ in state $\nu^{\prime}$ by enumerating the elements $x$ into full $e$-state $\left\langle e, \sigma^{\prime}, \tau\right\rangle$ and the elements $\hat{y}$ into $\left\langle e, \sigma^{\prime}, \tau\right\rangle$ since $\nu^{\prime} \geqq \nu$. Thus, we should try to arrange that $C$ covers $\hat{E}$ (and, in fact, every stream $\hat{X}$ ) and that $D \tau$ - 
exactly covers $\hat{E}$. Further we should enumerate $\hat{y}$ only into states $\nu$ which appear to be $\tau$-exactly covered by $D$. With this motivation, we now continue describing the construction.

At the beginning of a stage, there is at most one ball on the surface of one machine. If there is such a ball $x$, the stage consists of moving the ball according to the rules ( $\$ 4)$ down one track-a section of the surface between any two of the following: gates, doors, joins, or pockets. This may also cause us to enumerate $x \in$ $A, B, \hat{U}_{n}^{+}, \hat{V}_{n}^{+}$and to place other balls above holes. If there is no ball on the surface of the machine, we choose one ball above a hole and move it down the surface of the machine in successive stages. If at the beginning of a stage all balls are in pockets, we enumerate another element according to $g$. This may cause us to place some balls above holes.

ConstruCtion. Stage 0. Do nothing.

Stage $s+1$. The action consists of three steps.

Step 1. Adopt the first case below which holds.

Case 1. Some $x(\widehat{x})$ is on the surface of $M(\hat{M})$. There will be at most one such ball $x(\hat{x})$. Exactly one of the rules of $\S 4$ will apply to $x(\hat{x})$. Move $x(\hat{x})$ according to that rule.

Case 2. Some $x(\hat{x})$ is above a hole. Choose the least such $x$ (or least such $\hat{x}$ if $x$ doesn't exist) and place $x(\hat{x})$ on the surface of the machine at the end of the next track downward from the hole. (And so $x(\hat{x})$ will fall in Case 1 at the next stage.)

Case 3. Each $x(\hat{x})$ in $M(\hat{M})$ at stage $s+1$ is in a pocket. Enumerate one more value of our simultaneous enumeration $g$ and adopt the corresponding subcase below.

(a) $g$ enumerates $x \in W_{0}\left(x \in \hat{W}_{0}\right)$. Place a ball marked $x(\hat{x})$ above hole $H_{1}\left(\hat{H}_{1}\right)$ unless $x(\hat{x})$ is in Pocket $A(\hat{A})$. Enumerate $x \in$ $U_{0}\left(V_{0}\right)$.

(b) $g$ enumerates $x \in W_{e}\left(x \in \hat{W}_{e}\right), e>0$. By the convention on the enumeration of $W_{0}\left(\hat{W}_{0}\right)$ and Subcase $3(\mathrm{a}), x(\hat{x})$ must be in some pocket of $M(\hat{M})$. Enumerate $x(\hat{x})$ in $U_{e}\left(V_{e}\right)$ unless $x(\hat{x})$ is in pocket $P$. If $x(\widehat{x})$ is in pocket $P$, Rule $R_{10}\left(\hat{R}_{10}\right)$ will apply to $x(\widehat{x})$. If $x(\hat{x})$ is in pocket $A(\hat{A})$ proceed to stage $s+2$. If $x(\hat{x})$ is in pocket $Q(\hat{Q})$, Rule $R_{8}\left(\hat{R}_{8}\right)$ may apply to $x(\widehat{x})$ and should be followed.

(c) $g$ enumerates some computation $\varphi_{i}(j)=k, i \leqq j$. Let $a_{1}^{s}<a_{2}^{s}<\cdots$ be the elements of $\bar{A}_{s}$ in increasing order. If $a_{j}^{s} \leqq k$, let $m^{\prime}$ be the least $m$ such that $a_{m}^{s}>k$. Remove those balls marked 
$a_{j}^{s}, a_{j+1}^{s}, \cdots, a_{m^{\prime}-1}^{s}$ from pockets $P$ and $Q$ or from the store of balls not yet in $M$ and place them above hole $H_{4}$. (This may cause various other balls to be removed from pockets $P(\hat{P})$ according to the rules.)

Step 2. Apply Rule $R_{4}$ to every $x$ in pocket $Q$ in increasing order of $x$. Apply Rule $\hat{R}_{4}$ to every $\hat{x}$ in pocket $\hat{Q}$ in increasing order of $x$.

Step 3. Apply Rule $R_{11}\left(\hat{R}_{11}\right)$ to every element in pocket $P(\hat{P})$.

4. The rules. We now give the rules and some of the properties of the construction. Rules with numbered subscripts are the same or substantially the same as the corresponding rule in the proof of the Extension Theorem [5, Theorem 2.2]. Rules with lettered subscripts are new to this construction.

Rule $R_{1}\left(\hat{R}_{1}\right)$ determines which balls enter tracks $C_{1}$ and $C_{2}\left(\hat{C}_{1}\right.$ and $\widehat{C}_{2}$ ) when they reach door $D_{1}$. We let $\mathscr{R}_{s}$ denote a certain sequence defined by induction on $s$ and containing (exactly once) each pair $\langle\nu, j\rangle$ for all $j \in\{1,2\}$ and all full $e$-states $\nu$, for $e<s$. Let $\mathscr{R}_{0}=\left\{\left\langle\nu_{-1}, 1\right\rangle,\left\langle\nu_{-1}, 2\right\rangle\right\}$.

RULE $R_{1}$. Suppose that sequence $\mathscr{R}_{s}$ is given. If an element $x$ enters track $C$ at stage $s$, then at stage $s+1$ it enters either track $C_{1}$ or $C_{2}$ (with $\nu(s+1, x, x)=\nu(s, x, x)$ ) as follows. Let $\left\langle\nu^{\prime}, i^{\prime}\right\rangle$ be the first pair $\langle\nu, i\rangle$ on the sequence $\mathscr{R}_{s}$ such that $\nu \lessgtr \nu(s, x, x)$. Remove $\left(\nu^{\prime}, i^{\prime}\right\rangle$ from its present position on $\mathscr{R}_{s}$, place it at the end of the sequence, and place $x$ on track $C_{i^{\prime}}$. In this case we say that $\left\langle\nu^{\prime}, i^{\prime}\right\rangle$ is reset at stage $s+1$. Finally, whether an element $x$ entered track $C$ or not, add $\langle\nu, i\rangle$ at the end of the sequence (in any fixed effective order) for each $i \in\{1,2\}$ and each full $s$-state $\nu$. Let $\mathscr{R}_{s+1}$ denote the resulting sequence.

RULE $\hat{R}_{1}$. Like $R_{1}$ but with $\hat{C}, \hat{C}_{1}, \widehat{C}_{2}$ in place of $C, C_{1}, C_{2}$.

Rule $R_{A}\left(\hat{R}_{A}\right)$ is similar and determines which balls enter tracks $C_{5}$ and $C_{6}\left(\hat{C}_{5}\right.$ and $\left.\widehat{C}_{6}\right)$ when they reach door $D_{3}$.

RULE $R_{A}$. Let $\mathscr{R}_{s}^{\prime}$ be a sequence of full $e$-states with the same properties as $\mathscr{R}_{s}$ in Rule $R_{1}$ except that $C, C_{1}$, and $C_{2}$ are replaced by $C_{2}, C_{5}$, and $C_{8}$. Rule $R_{A}$ reads as Rule $R_{1}$, but with $\mathscr{R}^{\prime}, C_{5}, C_{6}, C_{2}$ replacing $R, C_{1}, C_{2}, C$.

RULE $\hat{R}_{A}$. Like Rule $R_{A}$ but with $\hat{C}_{2}, \hat{C}_{5}$, and $\hat{C}_{6}$ replacing $C_{2}, C_{5}$, and $C_{6}$. 
Lemma 4.1. Streams $C_{1}, C_{2}, C_{5}, C_{6}\left(\hat{C}_{1}, \hat{C}_{2}, \hat{C}_{5}, \hat{C}_{8}\right)$ are each equivalent to $C(\widehat{C})$.

Proof. The proof follows by making the obvious modifications to [5, Lemma 4.1].

The next rules are new and concern gate $G_{2}$. If $X$ is a track, let $\mathscr{S}_{\leqq s}(X)$ be the concatenation of the $\mathscr{S}_{t}$ for $t \leqq s$. The purpose of track $C_{6}\left(\hat{C}_{6}\right)$ is to arrange for $A$ to cover $\hat{A}$ ( $\hat{A}$ to dual cover $A$ ) to fulfill the hypothesis of the Extension Theorem of Soare. We will check members of $\mathscr{S}_{\leqq s}\left(\widehat{C}_{9}\right)$ which are successfully covered. We must be careful to restrain our enumerations to make $B$ nonhypersimple and $A$ coinfinite.

RULE $R_{B}$. Suppose $x$ is on track $C_{B}$ at the beginning of stage $s+1$. If there is a full $e$-state $\nu$ on $\mathscr{S}_{s_{s}}\left(\widehat{C}_{9}\right)$ for some $e \leqq x$ which satisfies

(1) $\nu$ is unchecked,

(2) $\nu \leqq \nu(s, e, x)$;

place $x$ on track $C_{8}$ at stage $s+1$ and check all $\nu$ on $\mathscr{S}_{\leqq_{8}}\left(\widehat{C}_{9}\right)$ such that $\nu \leqq \nu(s, e, x)$. Otherwise, place $x$ on track $C_{7}$.

RULE $R_{\hat{B}}$. Suppose $\hat{x}$ is on track $\hat{C}_{b}$ at the beginning of stage $s+1$ and $x \in D_{h(e)}$. If there is a full $i$-state $\nu$ on $\mathscr{S}_{\leqq s}\left(C_{9}\right)$ for some $i \leqq x$ which satisfies

(1) $\nu$ is unchecked,

(2) $\nu(s, i, \hat{x}) \leqq \nu$,

(3) the unchecked $\nu$ is one of the first $e$ members of $\mathscr{S}_{\leqq s}\left(C_{9}\right)$; place $\hat{x}$ on track $\hat{C}_{8}$ at stage $s+1$ and check all $\nu$ on $\mathscr{S}_{\leqq_{s}}\left(C_{9}\right)$ such that $\nu(s, i, \hat{x}) \leqq \nu$. Otherwise, place $\hat{x}$ on track $\hat{C}_{7}$.

Let $A(B)$ be the set consisting of those elements $x(\hat{x})$ which reside in pocket $A(\hat{A})$ at the end of the construction. The following rules clearly guarantee that $A$ and $B$ are r.e.

RULE $R_{C}\left(\hat{R}_{C}\right)$. No $x(\widehat{x})$ may leave pocket $A(\hat{A})$.

As described previously, the pocket $\hat{Q}(Q)$ will finally contain the elements of the domain of a finite-one map with the elements of $P(\hat{P})$ as range. The desired permutation $p$ will be determined by this map. Rule $R_{2}$ governs the entry of elements into the pocket $P$. Roughly, an element enters pocket $P$ if it is an appropriate "mate" for an element (or finite set of elements) of $\hat{Q}$, or if it is a more "desirable" mate for an element of $\hat{Q}$ than the present mate. 
Our Rule $\hat{R}_{2}$ is the same as Soare's, but Rule $R_{2}$ needs considerable modification. Roughly this is because now elements can leave pocket $P$ to meet the positive requirements on $A$. Before stating Rule $R_{2}$, we introduce some notation.

In Definition 4.2 , we define uniformly recursively in $s$ a sequence $\mathscr{K}_{s}^{s}$ of full states which contains each full $j$-state, $j<s$, exactly once. (This is the same definition that Soare gives.)

DEFINITION 4.2. (a) If $\nu_{1}, \nu_{2} \in \mathscr{K}_{s}$, we write $\nu_{1} \leqq_{s}^{*} \nu_{2}$ if $\nu_{1}$ precedes $\nu_{2}$ in the sequence $\mathscr{K}_{s}$.

(b) Let $\mathscr{K}_{0}=\left\{\nu_{-1}\right\}$. Given $\mathscr{K}_{s}$, let $\mathscr{K}_{s+1}^{2}=\left\{\nu: \nu \in \mathscr{K}_{s}\right.$ and $\left(\exists \nu^{\prime}\right)$ $\left[\nu<^{\tau} \nu^{\prime}\right.$ and $\left.\left.\nu^{\prime} \in \mathscr{S}_{s+1}(C) \cup \mathscr{S}_{s+1}(D)\right]\right\}$. Let $\mathscr{K}_{s+1}^{1}=\mathscr{K}_{s}-\mathscr{K}_{s+1}^{2}$. Let $\mathscr{K}_{s+1}^{3}$ be a sequence of all full $s$-states arranged in some effective order (uniformly in $s$ ) such that $\nu^{\prime}=\left\langle s, \sigma^{\prime}, \tau^{\prime}\right\rangle$ precedes $\nu=\langle s, \sigma, \tau\rangle$ if $\tau^{\prime} \varsubsetneqq \tau$ or if $\tau=\tau^{\prime}$ and $\sigma \leqq \sigma^{\prime}$. Let $\mathscr{K}_{s+1}$ denote the concatenation of $\mathscr{K}_{s+1}^{1}, \mathscr{K}_{s+1}^{2}, \mathscr{K}_{s+1}^{3}$ in that order where $\mathscr{K}_{s+1}^{1}, \mathscr{K}_{s+1}^{2}$ have the orderings induced by $\varliminf_{*}^{*}$.

(c) Define a function $r(s, \nu)$ for $s \in N$ and $\nu \in K_{s}$ as follows:

$$
\begin{aligned}
& r\left(0, \nu_{-1}\right)=1, r(0, \nu) \text { is undefined if } \nu \neq \nu_{-1} ; \\
& r(s+1, \nu)= r(s, \nu) \text { if } \nu \in \mathscr{K}_{s+1}^{1} ; \\
& r(s+1, \nu)= \max \{r(s, \nu): r(s, \nu) \text { is defined }\}+k \text { if } \\
& \nu \in \mathscr{K}_{s+1}^{2} \cup \mathscr{K}_{s+1}^{3} \text { and is the } k \text { th such } \\
& \text { in the ordering } \leqq_{s+1}^{*} ; \\
& r(s+1, \nu) \text { is undefined if } \nu \notin \mathscr{K}_{s+1}^{-} .
\end{aligned}
$$

(d) Let $\mathscr{\varkappa}_{\omega}$ denote those $\nu$ which are in $\mathscr{K}_{s+1}^{2}$ for only finitely many $s$.

Think of the list $\mathscr{K}_{s}$ as a list of full $j$-states, $j<s$, each occupying a row. At stage $s+1$, certain full $j$-states (those in $\mathscr{C}_{s+1}^{2}$ ) are reset - they are removed from the list and placed on "fresh" rows below the list $\mathscr{K}_{s}$. Also at stage $s+1$, the full $s$ states are added to $\mathscr{K}_{s+1}$ in fresh rows below $\mathscr{K}_{s+1}^{2}$. In this picture, $r(s, \nu)$ is the number of the row which $\nu$ occupies in $\mathscr{K}_{s}$. Note that $\nu \in \mathscr{K}_{\omega}^{\prime}$ iff $\lim _{s} r(s, \nu)$ exists. Also note that $r(s, \nu) \leqq r\left(s, \nu^{\prime}\right)$ iff $\nu \leqq \nu^{\sharp}$.

If $\mathscr{S}$ is a sequence of full $e$-states, we say that $\nu_{0}$ is maximal with respect to $\mathscr{S}$ if

$$
(\forall \nu)\left[\left[\nu \in \mathscr{S} \text { i.o. and } \nu_{0} \leqq^{\tau} \nu\right] \Longrightarrow \nu=\nu_{0}\right] \text {. }
$$

Soare proves the following lemmas:

Lemma 4.3 [5, Lemma 5.1]. For all e-states $\sigma, \sigma^{\prime}, \tau$ and all $s>e$, 


$$
\sigma^{\prime} \supseteqq \sigma \Rightarrow\left[\left\langle e, \sigma^{\prime}, \tau\right\rangle \leqq \leqq_{s}^{\sharp}\langle e, \sigma, \tau\rangle\right] .
$$

Lemma 4.4 [5, Lemma 5.2]. For all $\nu, \nu \in \mathscr{K}_{\omega}$ (and hence $\lim _{s} r(s, \nu)$ exists $)$ just if $\nu$ is maximal with respect to $\mathscr{S}(D)$.

Let $P_{s}, Q_{s}, M_{s}\left(\hat{P}_{s}, \hat{Q}_{s}, \hat{M}_{s}\right)$ denote the set of elements in pocket $P$, pocket $Q$, machine $M(\hat{P}, \hat{Q}, \hat{M})$ at the end of stage $s$.

Definition 4.5. Define (in increasing order of $\prec$ ) a function $\hat{q}(s, \nu)$ by $\hat{q}(s, \nu)=\mu \hat{y} \in \hat{Q}_{s}$ such that

(1) $\nu \precsim \nu(s, y, \widehat{y})$,

(2) $\hat{q}\left(s, \nu^{\prime}\right) \neq \hat{y}$ for any $\nu^{\prime} \prec \nu$.

If $\hat{y}$ does not exist, then $\hat{q}(s, \nu)$ is undefined.

Clearly, if $\hat{y} \in \hat{Q}_{s}, \hat{y}=\hat{q}(s, \nu)$ for some $\nu$. Roughly, the goal of Rule $R_{2}$ is to define a function $p(s, \nu)$ with values in $P_{s}$ so that if $\hat{q}(\nu)=\lim _{s} \hat{q}(s, \nu)$ and $p(\nu)=\lim _{s} p(s, \nu)$, then $\hat{q}(\nu) \rightarrow p(\nu)$ is the intended piece of the permutation mapping $\hat{Q}$ to $P$. The major difficulty is that $p(\nu)$ may not necessarily exist even if $\hat{q}(\nu)$ does. In this case, we will try to arrange that $\hat{q}\left(\nu^{\prime}\right)$ exists for only finitely many $\nu^{\prime}>\nu$, thereby enabling us to construct at least a finite-one map. Our major concern is to control the cases in which $p(s, \nu)$ becomes undefined infinitely often for a fixed $\nu$.

Definition 4.6. If $x \in M_{s}$ and $\nu$ is a full $e$-state such that $\nu \lesssim$ $\nu(s, x, x)$, the $\nu$-rank of $x$ at stage $s$, denoted $\rho(s, \nu, x)$, is the minimum of the set $\left\{r\left(s, \nu^{\prime}\right): \nu \precsim \nu^{\prime} \precsim \nu(s, x, x)\right\}$.

Rule $R_{2}$, which governs the selection of $p(s, \nu)$, will depend on the function $\rho$ defined above as well as certain markers $\Lambda_{\langle k, \ell, \nu\rangle}$. Rule $R_{2}$ will choose $p(s, \nu)$ so that the value $\rho(s, \nu, p(s, \nu))$ is as small as possible. (The reason for this measure of "desirability" of candidates for $p(s, \nu)$ is not obvious but will come out in the proof of Lemma 5.17.) The marker $\Lambda_{\langle k, \ell, \nu\rangle}$ will be used to prevent the positive requirements on $A$ from enumerating $p(s, \nu)$ into $A$ infinitely often where $k=\rho(s, \nu, p(s, \nu))$ and $\ell=(\mu t)\left(\forall \nu^{\prime} \precsim \nu\right)\left[\hat{q}\left(\zeta, \nu^{\prime}\right)=\hat{q}\left(\nu^{\prime}\right)\right]$.

Let $\nu_{k}$ be the unique $\nu$ such that $(\exists s)[r(s, \nu)=k]$. For each $k, \iota \in N$, form triples $\langle k, \iota, \nu\rangle$ where $\nu \precsim \nu_{k}$. Linearly order these triples by

$$
\begin{aligned}
& \langle k, \ell, \nu\rangle<\left\langle k^{\prime}, \iota^{\prime}, \nu^{\prime}\right\rangle \text { if } 2^{k} 3^{\iota}<2^{k^{\prime}} 3^{\iota^{\prime}} \text { or } \\
& k=k^{\prime}, \iota=\ell^{\prime} \text {, and } \nu \prec \nu^{\prime} .
\end{aligned}
$$

We will suppose that these triples have been put in 1-1 order preserving correspondence with $N$ and we will denote the integer cor- 
responding to $\langle k, \ell, \nu\rangle$ also by $\langle k, \ell, \nu\rangle$. When an element $x$ enters pocket $P$ (necessarily by Rule $R_{2}$ ) we will assign a certain marker $\Lambda_{\langle k, \ell, v\rangle}$ to it. That marker remains assigned to $x$ until $x$ leaves pocket $P$, at which time the assignment will be cancelled.

RULE $R_{2}$. Suppose that $x$ is on track $D$ at the end of stage s. Let $\nu^{\prime}$ be the first $\nu$ in the ordering $\lesssim$ such that

(1) $\nu \precsim \nu(s, x, x)$,

(2) $\hat{q}(s, \nu)$ is defined,

(3) either $p(s, \nu)$ is undefined or

$$
\rho(s, \nu, x)<\rho(s, \nu, p(s, \nu)) .
$$

If $\nu^{\prime}$ exists, then at stage $s+1$ place $x$ in pocket $P$, let $p\left(s+1, \nu^{\prime}\right)=$ $x$ (unless $x$ is immediately removed from pocket $P$ by the remainder of this Rule), and remove from $P$ all elements $p\left(s, \nu^{\prime \prime}\right)$ such that $\nu^{\prime} \precsim \nu^{\prime \prime}$ (and place these according to Rule $R_{12}$ ). Assign marker $\Lambda_{\left\langle k, \varepsilon, \nu^{\prime}\right\rangle}$ to $x$ where

$$
\begin{aligned}
k= & \rho\left(s, \nu^{\prime}, x\right) \text { and } \\
& \ell=(\mu t)(\forall z)_{t \leqq z \leqq s}\left(\forall \nu^{\prime \prime} \precsim \nu^{\prime}\right)\left[\hat{q}\left(s, \nu^{\prime \prime}\right)=\hat{q}\left(z, \nu^{\prime \prime}\right)\right] .
\end{aligned}
$$

Let $m$ be the integer such that $x=a_{m}^{s}$. If $m \leqq\left\langle k, \ell, \nu^{\prime}\right\rangle$ do nothing more, but if $\left\langle k, \ell, \nu^{\prime}\right\rangle\left\langle m\right.$, place above hole $H_{4}$ all $a_{i}^{s}$ where $\left\langle k, \ell, \nu^{\prime}\right\rangle \leqq$ $i<m$. (This may cause other balls to be removed from pocket $P$ by the next rule, Rule $R_{D}$.) If $\nu^{\prime}$ does not exist, place $x$ on track $D_{1}$.

RULE $R_{D}$. If $x=p(s, \nu)$ is placed above hole $H_{4}$ at stage $s+1$, remove each $p\left(s, \nu^{\prime}\right)$ for $\nu^{\prime} \succsim \nu$ and place these balls according to Rule $R_{12}$.

RULE $R_{E}$. A ball $x$ may only be placed above hole $H_{4}$ by Subcase 3(c) of Step 1 of the construction or Rule $R_{2}$.

Rule $\hat{R}_{2}$, the pockets $\hat{P}$ and $Q$, and the functions $\hat{p}$ and $q$ are duals to the above except that the markers $\Lambda_{\langle k, \ell, \nu\rangle}$ are omitted. This is because there are no positive requirements on $B$ requiring elements to be taken out of $\hat{P}$. Thus, there is no Rule $\hat{R}_{D}$, and $\hat{R}_{E}$ reads as follows:

RULE $R_{E}$. No $\widehat{x}$ may be placed above hole $\hat{H}_{4}$.

By induction on $s$, we now define recursively, uniformly in $s$, sequences of full $j$-states $\mathscr{M}_{s}, \mathscr{P}_{s}$ such that $\mathscr{M}_{s} \subseteq \mathscr{P}_{s} \subseteq \mathscr{K}_{s}$ for all $s$. Our main goal is to prove that $D$ exactly covers $\hat{Q}$ so that 
$\widehat{Q}$ sees enough "mates" to achieve the desired piece of the permutation. (Here $\hat{Q}$ can be considered a stream in the following sense: let $\mathscr{S}_{s+1}(\hat{Q})=\left\{\nu(s+1, e, \hat{x}): e \leqq x, \widehat{x} \in \hat{Q}_{s+1}-\hat{Q}_{s}\right.$ or $\hat{x} \in \hat{Q}_{s+1} \cap \hat{Q}_{s}$, and $\nu(+1, x, \hat{x}) \neq \nu(s, x, \hat{x})\}$.) We will do this by insuring that $C$ covers every stream $\hat{X}$ of $\hat{M}$ and that $D \tau$-exactly covers every stream that $C$ covers.

Definition 4.7. (a) Let $\mathscr{C}_{0}=\left\{\nu_{-1}\right\}$. Given $\mathscr{K}_{s+1}$ and $\mathscr{K}_{s}$ we will define $\mathscr{A}_{s+1}$. Let $\nu=\langle e, \sigma, \tau\rangle$ and suppose we have already determined whether $\nu^{\prime} \in \mathscr{M}_{s+1}$ if $\left|\nu^{\prime}\right|<e$. We say $\nu$ is excluded from $\mathscr{M}_{s+1}$ if one of the following conditions holds:

Condition 1. $\left(\exists \nu^{\prime}\right)\left[r\left(s, \nu^{\prime}\right)<r(s, \nu)\right.$ and $\left.\nu^{\prime} \in \mathscr{K}_{s+1}^{2}\right]$.

Condition 2. $\left(\exists \nu^{\prime}\right)(\exists \hat{X})\left[\nu^{\prime} \in \mathscr{S}_{s}(\hat{X})-\mathscr{P}_{s}\right.$ and $\left.\left|\nu^{\prime}\right|<e\right]$, where $\hat{X}$ is any stream of $\hat{M}$ except $\hat{A}$, including $\hat{Q}$.

Condition 3. There is a full $j$-state $\nu^{\prime}$ and an element $x$ such that

(i ) $x=p\left(s, \nu^{\prime}\right)$,

(ii) $x$ is placed above hole $H_{4}$ at stage $s+1$,

(iii) $\rho\left(s, \nu^{\prime}, x\right) \leqq r(s, \nu)$,

(iv) $r(s, \nu) \geqq \ell=(\mu t \leqq s)(\forall z)_{t \leqq z \leqq s}\left(\forall \nu^{\prime \prime} \precsim \nu^{\prime}\right)\left[\hat{q}\left(z, \nu^{\prime \prime}\right)=\hat{q}\left(s, \nu^{\prime \prime}\right)\right]$.

Define $\nu \in \mathscr{M}_{s+1}$ if $\nu$ is not excluded from $\mathscr{M}_{s+1}$ and either $\nu \in \mathscr{M}_{s}$ or $\nu \in \mathscr{S}_{s+1}(D)$.

(b) Let $\mathscr{P}_{8+1}=\left\{\nu:\left(\exists \nu^{\prime}\right)\left[\nu^{\prime} \in \mathscr{C}_{s+1}\right.\right.$ and $\left.\left.\nu \varliminf^{\tau} \nu^{\prime}\right]\right\}$.

(c) Let $\mathscr{M}_{\omega}=\left\{\nu: \nu \in \mathscr{M}_{s}\right.$ for almost all $\left.s\right\}$.

(d) Let $\mathscr{P}_{\omega}=\left\{\nu: \nu \in \mathscr{P}_{s}\right.$ for almost all $\left.s\right\}$.

(e) Let $s_{\hat{x}}=\mu t\left[\hat{x} \in \hat{M}_{t}\right]$. Define $d(s, \hat{x})=\max \left\{e: \nu(s, e, \hat{x}) \in \mathscr{P}_{s}\right.$ and $\left.(\forall t)\left[s_{\hat{x}} \leqq t<s=e \leqq d(t, \hat{x})\right]\right\}$. For other $\hat{x}$ and $s$ let $d(s, \hat{x})=-1$. (Note that $\lim _{s} d(s, \hat{x})$ exists.)

(f) Let $\nu^{*}(s, \hat{x})=\left[\nu(s, x, \hat{x}]_{d(s, \hat{x})}\right.$ and $\nu^{*}(\hat{x})=\lim _{s} \nu^{*}(s, \hat{x})$.

We now give some intuition as to these lists. First, $\mathscr{K}_{\omega}$ is simply the maximal full $e$-states with respect to $\mathscr{S}(D)$. $\mathscr{M}_{\omega}$, except for the complications of conditions (2) and (3) of Definition 4.7(a), is an approximation to those states in $\mathscr{K}_{\omega}$ which appear infinitely often on $\mathscr{S}(D)$. Thus $\mathscr{P}_{\omega}$ is a recursive approximation to those full $e$-states that $D \tau$-exactly covers. If we knew the full $e$-states that $D$ does in fact $\tau$-exactly cover, we would be able to guarantee that $D \tau$-exactly covered $\hat{Q}_{\omega}$ by only enumerating elements of $\hat{M}$ into these states. As it is, $\nu^{*}(s, \hat{x})$ is simply a "guess" as to the largest initial segment of $\nu(s, x, \widehat{x})$ which $D \tau$-exactly covers.

The next rule, Rule $R_{3}$, determines what happens when an element $x$ arrives at gate $G_{1}$. Here the element $x$ may be enumerated 
in certain sets $\hat{V}_{n}^{+}$. The purpose of this rule is to insure that $D$ $\tau$-exactly covers any stream $\hat{X}$ which $C$ covers. In the next rule and associated lemmas, $X(\hat{X})$ ranges over streams of $M(\hat{M})$ except for $A(\hat{A})$.

Rule $R_{3}$ involves a certain r.e. sequence $\mathscr{C}$ of full $e$-states, which is the concatenation of the finite sequences $\mathscr{Y}_{s}, s \in N$, defined as follows. $\mathscr{C}_{s}=\phi$ unless there is some track $\hat{X}$ of $\hat{M}$, some element $\hat{y}_{1}$ on track $\hat{X}$ at stage $s$, and some full $e_{1}$-state $\nu_{1}$ such that

$$
\nu_{1} \in \mathscr{S}_{s}(\hat{X})-\mathscr{P}_{s}
$$

via $\widehat{y}_{1}$, in which case $\mathscr{H}_{s}$ consists of the following full $e_{1}$-states for each such $\hat{y}_{1}$ (in some effective order uniformly in $s$ ):

$$
\left\{\nu:(\exists t)_{\leqq}\left[\hat{y}_{1} \in \hat{M}_{t} \text { and } \nu\left(e_{1}, t, \hat{y}_{1}\right) \leqq^{\tau} \nu\right]\right\} \text {. }
$$

Once added to $\mathscr{H}$, a given $\nu$ is never removed from $\mathscr{H}$ or altered in position, although it may later be checked during an application of Rule $R_{3}$. Let $\mathscr{H}_{\leqq s}$ denote the sequence of elements added to $\mathscr{C}$ by the end of stage $s$.

RULE $R_{3}$. Suppose an element $x$ enters track $C_{1}$ at stage $s$. Let $\nu_{0}=\left\langle x, \sigma_{0}, \tau_{0}\right\rangle$ denote $\nu(s, x, x)$. Let $\nu_{1}=\left\langle e_{1}, \sigma_{1}, \tau_{1}\right\rangle$ be the first member $\nu=\langle e, \sigma, \tau\rangle \in \mathscr{H}_{\leqq s}$ such that:

(i) $\nu$ has not been checked by the end of stage $s$;

(ii) $e \leqq x$; and

(iii) $\nu \varliminf^{\sigma}\left[\nu_{0}\right]_{e}$ (i.e., $\sigma=\left[\sigma_{0}\right]_{e}$, and $\tau \supseteqq\left[\tau_{0}\right]_{e}$ ). If $\nu_{1}$ exists, then at stage $s+1$ check $\nu_{1}$, enumerate $x$ in $\widehat{V}_{n, s+1}^{+}$for each $n \leqq e_{1}$ such that $n \in \tau_{1}-\tau_{0}$ (so that $\nu\left(s+1, e_{1}, x\right)=\nu_{1}$ ), and place $x$ on track $C_{3}$. If $\nu_{1}$ fails to exist, then at stage $s+1$ place $x$ on track $C_{4}$, and let $\nu(s+1, x, x)=\nu(s, x, x)$.

RULE $\hat{R}_{3}$. Same as Rule $R_{3}$, but with $C_{1}, C_{3}, C_{4}, \hat{C}, \hat{V}_{n}^{+}$, $\mathscr{Y}$ replaced by $\hat{C}_{1}, \hat{C}_{3}, \hat{C}_{4}, C, \hat{U}_{n}^{+}$, and $\mathscr{K}^{\prime}$ (which is defined in the analogous way using $\mathscr{P}_{s}^{\prime}$ and $\mathscr{S}_{s}(X)$ ), and with the roles of $\sigma$ and $\tau$ interchanged.

Rule $\hat{R}_{4}$ determines the enumeration of elements $\hat{x}$ in sets $\hat{U}_{n}^{+}$ while $\hat{x}$ is in pocket $\hat{Q}$. It is a combination of the Rules $\hat{R}_{4}$ and $\hat{R}_{5}$ of [5]. Thus there is no Rule $\hat{R}_{5}$ although we have kept the same numbering of the other rules to avoid confusion.

Rule $\hat{R}_{4}$. Suupose that $\hat{x}$ is in $\hat{Q}$ at the end of step 1 of stage $s+1$ of the construction. Then $\hat{x}$ remains in pocket $\hat{Q}$ through 
the end of stage $s+1$ and $\tau(s+1, \widehat{x})=\tau(s, \hat{x})$, (else $\widehat{x}$ was removed at stage $s+1$, step 1 , under Rule $\hat{R}_{8}$ ).

Case 1. If $\nu^{*}(s, \hat{x}) \in \mathscr{M}_{s}$ do nothing.

Case 2. Otherwise perform the following enumeration on $\hat{x}$. Let $\mathscr{S}_{s t}(D)$ denote the sequence which is the concatenation of the sequences $\left\{\mathscr{S}_{u}(D): u \leqq t\right\}$. Let $\nu^{\prime}(s+1)=\left\langle e, \sigma^{\prime}, \tau^{\prime}\right\rangle$ denote $\nu^{*}(s, \hat{x})$. Define

$$
\mathscr{F}_{s+1}=\left\{\nu: \nu \in \mathscr{C}_{s} \text { and } \nu^{\prime}(s+1) \leqq^{\tau} \nu\right\} .
$$

Note that $\nu^{\prime}(s+1) \in \mathscr{P}_{s}$ and thus $\mathscr{F}_{s+1} \neq \phi$, by the definitions of $\mathscr{P}_{s}$ and $d$. Furthermore, for each $\nu \in \mathscr{C}_{s}, \nu \in \mathscr{S}_{\leqq(s)}(D)$. Define $\nu^{\prime \prime}(s+1)=$ $\left\langle e, \sigma^{\prime \prime}, \tau^{\prime}\right\rangle$ to be the last $\nu$ on the sequence $\mathscr{S}_{\leqq(s)}(D)$ such that $\nu \in \mathscr{F}_{s+1}$. Enumerate $\hat{x}$ in $\hat{U}_{n, s+1}^{+}$for each $n \in \sigma^{\prime \prime}-\sigma^{\prime}$.

LEMMA 4.8. $(\forall \hat{x})\left[\hat{x} \in \hat{Q}_{\omega} \Rightarrow \nu^{*}(\widehat{x}) \in \mathscr{L}_{\omega}\right]$.

Proof. Let $e=\lim _{s} d(s, \hat{x})$. Let $\nu=\lim _{s} \nu(s, e, \hat{x})$. Then $\nu=$ $\nu^{*}(\hat{x})$. Let $s_{0}$ be any stage such that, for $s \geqq s_{0}, d(s, \hat{x})=e, \nu(s, e, x)=$ $\nu$, and $\hat{x} \in \hat{Q}_{s}$. By Rule $\hat{R}_{4}$, if $\nu \notin \mathscr{C}_{s}$ for any such $s, \hat{x}$ is enumerated in certain r.e. sets $\hat{U}_{n, s+1}^{+}$for $n \leqq e$ causing $\nu(s+1, e, x) \neq \nu$, contrary to the hypothesis on stage $s_{0}$. Thus $\nu=\nu^{*}(x) \in \mathscr{M}_{s}$ for each $s \geqq s_{0}$.

Rules $R_{4}$ and a function $\hat{d}$ are similar and are omitted.

We now give the rest of the rules of the machines. The duals of these rules are similar and are omitted.

RULE $R_{6}$. An element $x \in \hat{V}_{n, s+1}^{+}-\hat{V}_{n, s}^{+}$only if at stage $s+1$, Rule $R_{3}$ or $R_{4}$ applies to $x$.

RULE $R_{7}$. If $x \in U_{n, s+1}-U_{n, s}$ and $x \in M_{s}$ then $x$ is in some pocket at the beginning of stage $s+1$.

RULE $R_{s}$. If $x \in Q_{s}$ and $x \in U_{n, s+1}-U_{n, s}$ for some $n \leqq x$ remove $x$ from pocket $Q$ at stage $s+1$ and place $x$ above hole $H_{2}$.

RULE $R_{8}$. If $x \in Q_{s}$ then $x \in Q_{s+1}$ unless $x$ is removed at stage $s+1$ from pocket $Q$ under Rules $R_{2}$ or $R_{3}$ or Subcase $3(\mathrm{c})$ of the construction.

RULE $R_{10}$. If $x=p(s, \nu) \in P_{s}$ for some $\nu$ such that $|\nu|=e$ and $x$ is enumerated in $W_{n}$ at stage $s+1$ for some $n \leqq e$, then at stage $s+1$ remove from pocket $P$ all $p\left(s, \nu^{\prime}\right)$ such that $\nu \precsim \nu^{\prime}$ and enumer- 
ate $x$ in $U_{n}$. If $x$ is enumerated in $W_{n}$ for some $n>e$, proceed to stage $s+2$.

RULE $R_{11}$. If $\hat{q}(s, \nu) \neq \hat{q}(s+1, \nu)$ or $\hat{q}(s+1, \nu)$ is undefined, then remove from pocket $P$ at stage $s+1$ all $p\left(s, \nu^{\prime}\right)$ such that $\nu \lesssim \nu^{\prime}$.

RULE $R_{12}$. If $x \in P_{s}$, then $x \in P_{s+1}$ unless $x$ is removed from $P$ at stage $s+1$ by the construction Subcase $3(\mathrm{c})$, or Rules $R_{2}, R_{10}$, $R_{11}$, or $R_{D}$. If $x$ is not placed above hole $H_{4}$ by Rule $R_{2}$ or Subcase $3(c)$ and $x$ last entered pocket $P$ at stage $t$, then place $x$ above hole $H_{2}$ if $\nu(s+1, x, x) \neq \nu(t, x, x)$ and place $x$ above hole $H_{3}$ otherwise.

RULE $R_{F}$. If at the end of stage $s, x$ is on any of the tracks $C_{0}, C_{4}, C_{5}, C_{7}, C_{8}, D_{1}$, or $E$, place $x$ at stage $s+1$ on the next track or pocket in the downward direction (the direction of the arrows).

5. The verification. The first two lemmas of this section guarantee that each ball eventually reaches a pocket where it resides for the rest of the construction.

LEMmA 5.1. Each element $x(\hat{x})$ re-enters the surface of $M(\hat{M})$ at most finitely often.

Proof. Suppose $x$ is an element of $M$. (The case $\hat{x} \in \hat{M}$ is similar.) From hole $H_{4}, x$ can only reach pocket $A$ and so $x$ may be placed above hole $H_{4}$ at most once. Suppose $x$ is placed above hole $H_{2}$ at stage $s+1$. Then $x \in P_{s}$ or $Q_{s}$. If $x \in Q_{s}-Q_{s+1}$ then $\nu(s+1, x, x) \neq \nu(s, x, x)$ by Rule $R_{8}$, which can happen only finitely often. Similarly, if $x \in P_{s}-P_{s+1}, \nu(s+1, x, x) \neq \nu(t, x, x)$ by Rule $R_{12}$ where $t$ is the last stage before $s$ that $x$ entered $P$. Thus $x$ can be placed above hole $H_{2}$ only finitely often. If $x$ is placed above hole $H_{3}, x$ can only re-enter $M$. from pocket $Q$ and hence via hole $H_{2}$.

LEMMA 5.2. Each element $x(\hat{x})$ is in some particular pocket for cofinitely many stages.

Proof. By Lemma 5.1, each element $x$ re-enters only finitely often. Once $x$ is on the surface of $M$ it will reach a pocket by the construction. The only possible obstacle then is for $x$ to remain above a hole cofinitely often. Choose any stage $s$ such that $x$ is above a hole. At stage $s$, only finitely many elements are in $M_{s}$. By Lemma 5.1, each of the elements besides $x$ can generate only 
finitely many moves; eventually $x$ will be the $x$ of Case 2 of step 1 of the construction and will re-enter the surface of $M$.

We can actually prove already that $B$ is not hypersimple.

LEMMA 5.3. $B$ is not hypersimple.

Proof. That

$B=\{x: \hat{x}$ is in pocket $B$ for cofinitely many stages $\}$

is r.e. is already clear by Rule $\hat{R}_{C}$. By Rule $\hat{R}_{E}$, no $\hat{x}$ enters pocket $B$ except by track $\hat{C}_{6}$ and hence by Rule $\hat{R}_{B}$. Since each $\hat{x} \in D_{h(e)}$ which enters $B$ in this way checks one of the first $e$ members of $\mathscr{S}_{S_{B}}\left(C_{\theta}\right),\left|D_{h(e)} \cap B\right| \leqq e$. Of course $\left\{D_{h(e)}\right\}_{e \in N}$ witnesses that $B$ is not hypersimple since $\left|D_{h(e)}\right|=e+1 \Rightarrow D_{h(e)} \cap \bar{B} \neq \phi$.

The next set of lemmas together will show that $D \tau$-exactly covers every stream $\hat{X}$ of $\hat{M}$. Recall that markers $\Lambda_{\langle k, \ell, \nu\rangle}$ are assigned to elements of pocket $P$ so that each element of pocket $P$ has exactly one marker assigned to it at stage $s$. Marker $\Lambda_{\langle k, \ell, \nu\rangle}$ was supposed to insure that if $\iota=(\mu t)\left(\forall \nu^{\prime} \precsim \nu\right)\left[\hat{q}\left(t, \nu^{\prime}\right)=\hat{q}\left(\nu^{\prime}\right)\right]$ and $\rho(s, \nu, p(s, \nu))=k$ for infinitely many $s$, then the positive requirements on $A$ do not remove $p(s, \nu)$ from pocket $P$ infinitely often. We arranged this by guaranteeing that, if $x=p(s, \nu)$ has marker $\Lambda_{\langle k, \ell, \nu\rangle}$ assigned to it at stage $s$ and $\langle k, \ell, \nu\rangle=m$, then $x \leqq a_{m}^{s}$. Thus only computations $\varphi_{i}(j)$ for $i, j \leqq m$ can put such an $x$ in $A$. Lemma 5.5 guarantees that the use of marker $\Lambda_{\langle k, \ell, \nu\rangle}$ accomplishes somewhat more than the above. This lemma depends heavily on the careful indexing of triples $\langle k, \iota, \nu\rangle$. We first prove a technical lemma which will be used in Lemma 5.5 .

Lemma 5.4. Suppose that at stage $s+1 x$ is placed above hole $H_{2}$. Then $x$ is placed on track $C$ at some stage $t>s+1$.

Proof. By the construction, $x$ eventually enters track $C$ unless $x$ is placed above hole $H_{4}$ at some stage $t+1>s+1$. By Rule $R_{E}, x$ may only be placed above hole $H_{4}$ by Subcase $3(\mathrm{c})$ of the construction or by Rule $R_{2}$. Subcase $3(\mathrm{c})$ of the construction never applies while $x$ is above hole $H_{2}$. If Rule $R_{2}$ placed $x$ above hole $H_{4}$ at stage $t+1$, then at stage $t$ some $y$ is on track $D$ and $x<y$. But, by the order in which balls are released from holes, no $y>x$ is ever on any track while $x$ is above hole $H_{2}$. (This can be proved easily by induction on the stages that $x$ is above hole $H_{2}$ ).

Lemma 5.5. Fix $k, \ell, \nu$. Then there are only finitely many 
stages $s+1$ such that Rule $R_{2}$ assigns marker $\Lambda_{\langle k, \ell, \nu\rangle}$ at stage $s+1$.

Proof. By induction on $m=\langle k, \ell, \nu\rangle$, we may assume that there is a stage $s_{0}$ such that if $n=\left\langle k^{\prime}, \ell^{\prime}, \nu^{\prime}\right\rangle\left\langle m\right.$, then Rule $R_{2}$ never assigns marker $\Lambda_{n}$ at a stage $s+1 \geqq s_{0}$ and, if the last assignment of marker $A_{n}$ is ever cancelled, it is cancelled before stage $s_{0}$. Of course we may also assume that Subcase 3(c) of step 1 of the construction never applies to $a_{m}^{s}$ if $s \geqq s_{0}$. Let $\nu_{k}$ denote the unique $\nu$ such that $r(s, \nu)=k$ for some $s$. If Rule $R_{2}$ assigns $\Lambda_{\langle k, \ell, \nu\rangle}$ to $x$ at stage $s+1$, then

(1) $x=p(s+1, \nu)$,

(2) $k=\rho(s, \nu, x)$,

(3) $\ell=(\mu t \leqq s)(\forall z)_{t \leqq s \leqq s}\left(\forall \nu^{\prime} \lesssim \nu\right)\left[\hat{q}\left(z, \nu^{\prime}\right)=\hat{q}\left(s, \nu^{\prime}\right)\right]$,

(4) $x \leqq a_{m}^{s+1}$.

For the sake of a contradiction, assume that Rule $R_{2}$ assigns $\Lambda_{\langle k, \ell, \nu\rangle}$ at infinitely many stages $s+1>s_{0}$. Thus we also may assume that $\lim _{s} v\left(s, \nu_{k}\right)=k$ since $\rho(s, \nu, x)=k$ for any stage $s$ such that $\Lambda_{\langle k, \ell, \nu\rangle}$ is assigned at stage $s+1$ and $\rho(s, \nu, x)=r\left(s, \nu^{\prime}\right)$ for some $\nu^{\prime} \gtrsim \nu$. Further, we may assume that $\hat{q}(\nu)=\lim _{s} \hat{q}(s, \nu)$ exists by (3). At a stage $s+1>s_{0}$ such that $\Lambda_{\langle k, \ell, \nu\rangle}$ is assigned by Rule $R_{2}$ at stage $s+1$ the element $x$ which newly arrives in pocket $P$ has full $x$-state satisfying $\nu \precsim \nu_{k} \precsim \nu(s, x, x)$. Since this element was not preferred as $p\left(s+1, \nu^{\prime}\right)$ for any $\nu^{\prime} \prec \nu$, it must be the case that $\rho\left(s, \nu^{\prime}, p\left(s, \nu^{\prime}\right)\right) \leqq k$ for any such $\nu^{\prime} \prec \nu$. Now we note that $\rho\left(s, \nu^{\prime}, p\left(s, \nu^{\prime}\right)\right)$ does not decrease as long as $p(s, \nu)$ remains in pocket $P$ since $p\left(s, \nu^{\prime}\right)$ is not enumerated in any r.e. set while in pocket $P$. Thus, at stage $s, p\left(s, \nu^{\prime}\right)$ had some marker $\Lambda_{\left\langle k^{\prime}, \ell^{\prime}, \nu^{\prime}\right\rangle}$ still assigned to it where $k^{\prime} \leqq \rho\left(s, \nu^{\prime}, p\left(s, \nu^{\prime}\right)\right) \leqq k$ and $\ell^{\prime} \leqq \ell$ (since $\left.\nu^{\prime} \prec \nu\right)$. Thus $\left\langle k^{\prime}, \ell^{\prime}, \nu^{\prime}\right\rangle\left\langle\langle k, \ell, \nu\rangle\right.$ so that, by our induction hypothesis $p\left(s_{0}, \nu^{\prime}\right)=$ $p\left(\nu^{\prime}\right)$ because $\Lambda_{\left\langle k^{\prime}, \ell^{\prime}, \nu^{\prime}\right\rangle}$ is never assigned by Rule $R_{2}$ or cancelled after stage $s_{0}$.

Suppose that the assignment of $\Lambda_{m}$ is cancelled at some stage $s+1>s_{0}$. Then, by Rule $R_{12}, p(s, \nu)$ is taken out of pocket $P$ at stage $s+1$ by Rules $R_{2}, R_{10}, R_{11}$, or $R_{D}$ or by Subcase $3(\mathrm{c})$ of the construction. It is easy to see that $p(s, \nu)$ could not be removed by Rule $R_{11}$ or Subcase $3(\mathrm{c})$ of the construction by our hypotheses about stage $s_{0}$. Also Rule $R_{D}$ could not remove $p(s, \nu)$ at stage $s+1$ because this would require some $p\left(s, \nu^{\prime}\right)$ to be placed above hole $H_{4}$ at stage $s+1$ for some $\nu^{\prime} \prec \nu$. If Rule $R_{2}$ removes $p(s, \nu)$ at stage $s+1$, then either Rule $R_{2}$ defines $p(s+1, \nu)$, Rule $R_{2}$ defines $p\left(s+1, \nu^{\prime}\right)$ for some $\nu^{\prime} \prec \nu$, or Rule $R_{2}$ assigns a marker $\Lambda_{n}$ for $n<m$. The latter two cases cannot happen after stage $s_{0}$, so suppose Rule $R_{2}$ defines $p(s+1, \nu)$. Then $\rho(s, \nu, x)<\rho(s, \nu, p(s, \nu))$ for some $x$ on track $D$ at stage $s$. But $\rho(s, \nu, p(s, \nu))=k$ since 
$p(s, \nu)$ does not change state while in $P$ nor is $\nu_{k}$ ever reset. Thus Rule $R_{2}$ assigns a marker $\Lambda_{\left\langle k^{\prime}, \ell, \nu\right\rangle}$ to $x$ with $k^{\prime}<k$. This contradicts the hypothesis about $s_{0}$ since $\left\langle k^{\prime}, \ell, \nu\right\rangle\langle\langle k, \ell, \nu\rangle$. Finally, we consider Rule $R_{10}$. If $x=p(s, \nu)$ is removed from pocket $P$ at stage $s+1$ by Rule $R_{10}, x \in U_{n, s+1}-U_{n, s}$ for some $n \leqq|\nu| \leqq\left|\nu_{k}\right|$. By Rule $R_{12}, x$ is placed above hole $H_{2}$. By Lemma $5.4, x$ is placed on track $C$ at some stage $t+1>s+1$. Then $\nu_{k}<[\nu(t+1, x, x)]_{\nu_{k} \mid}$ and so, at stage $t+1, \nu_{k} \in \mathscr{K}_{t+1}^{2}$ and $r\left(t+1, \nu_{k}\right)>k$, contradicting the assumption that $\lim _{s} r\left(s, \nu_{k}\right)=k$. This final contradiction establishes the lemma.

Lemma 5.6. Any full e-state $\nu \in \mathscr{K}_{\omega}$ is excluded from $\mathscr{M}_{s+1}$ by condition 3 of Definition 4.7(a) only finitely often.

Proof. Let $k=\lim _{s} r(s, \nu)$ which exists since $\nu \in \mathscr{K}_{\omega}$. If $\nu$ is excluded by condition 3 at stage $s+1$, some element $x=p\left(s, \nu^{\prime}\right)$ is placed above hole $H_{4}$ at stage $s+1$ where $x$ was assigned a marker $\Lambda_{\left\langle k^{\prime}, \ell^{\prime}, \nu^{\prime}\right\rangle}$ at stage $s$ with $k^{\prime} \leqq k$ and $\ell^{\prime} \leqq k$. Since there only finitely many such markers, $\nu$ is excluded by condition 3 only finitely often because of Lemma 5.5 .

The preceding lemma says that condition 3 exclusion was not used too often. In Lemma 5.17 we will see that condition 3 exclusion is used often enough.

Recall that $\mathscr{C}_{\omega}$ was our approximation to those full $e$-states that are in $\mathscr{S}(D)$ infinitely often and are the maximal such $e$-states with respect to $\leqq$. The next lemma derives the crucial properties of $\mathscr{C}_{\omega}$ and $\mathscr{F}_{\omega}$ under a certain assumption that will be verified later (in Lemma 5.9).

Lemma 5.7. Suppose that $\nu$ is excluded only finitely often from $\mathscr{C}_{s+1}$ under condition 2 of Definition 4.7(a). Then

(1) $\nu \in \mathscr{K}_{\omega}$ and $\nu \in \mathscr{S}(D)$ i.o. $\Rightarrow \nu \in \mathscr{K}_{\omega}$, and

(2) $\nu \in \mathscr{P}_{s}$ i.o. $\Rightarrow \nu \in \mathscr{P}_{\omega}$.

Proof. Fix $\nu=\langle e, \sigma, \tau\rangle$ with $\nu \in \mathscr{K}_{\omega}$ and $\nu \in \mathscr{S}(D)$ i.o. Consider the conditions under which $\nu$ could be excluded from $\mathscr{C}_{s+1}$. Since $\nu \in \mathscr{K}_{\omega}, \nu$ is excluded by condition 1 only finitely often. By hypothesis, $\nu$ is excluded by condition 2 only finitely often. Finally, $\nu$ is excluded by condition 3 only finitely often by Lemma 5.6. Thus, $\nu \in \mathscr{C}_{\omega}$ since $\nu \in \mathscr{S}(D)$ i.o.

(2) This follows from (1) as in [5, Lemma 5.3, p. 107].

Lemma 5.8. With the same hypothesis as Lemma 5.7 for $\nu_{1}$ and 
$\nu_{2}$, let $\nu_{1}$ and $\nu_{2}$, be full e-states. Suppose that $D$-exactly covers $\nu_{1}$ and Rule $\hat{R}_{4}$ applies at infinitely many stages $s$ such that $\nu^{\prime}(s)=\nu_{1}$ and $\nu^{\prime \prime}(s)=\nu_{2}$. Then $D$-exactly covers $\nu_{2}$.

Proof. Fix $\nu_{1}=\left\langle e, \sigma_{1}, \tau_{1}\right\rangle$ and $\nu_{2}=\left\langle e, \sigma_{2}, \tau_{2}\right\rangle$ satisfying the hypotheses and assume that $D$ does not $\tau$-exactly cover $\nu_{2}$. There is a full $e$-state $\nu_{3} \in \mathscr{S}(D)$ i.o. with $\nu_{1} \leqq^{\tau} \nu_{3}$ and $\nu_{3} \in \mathscr{K}_{\omega}$ for $D$ $\tau$-exactly covers $\nu_{1}$. If $\nu_{3}$ is excluded from $\mathscr{C}_{s}$ under condition 2 of Definition 3.4.7(a), so is $\nu_{2}$ since $\left|\nu_{3}\right|=\left|\nu_{2}\right|=e$. Thus $\nu_{3}$ is excluded only finitely often from $\mathscr{C}_{s}$ under condition 2 . Thus, by Lemma 5.7(1), $\nu_{3} \in \mathscr{C}_{\omega}$. But since $\nu_{2} \notin \mathscr{S}(D)$ i.o. and $\nu_{3} \in \mathscr{S}(D)$ i.o., for almost all $s$ we must have that some occurence of $\nu_{3}$ follows the last occurence of $\nu_{2}$ on $\mathscr{S}_{\leqq_{3}}(D)$. Thus, for almost all $s, \nu_{3}$ is preferred to $\nu_{2}$ as $\nu^{\prime \prime}(s)$ when $\nu^{\prime}(s)=\nu_{1}$.

Of course, Lemmas 5.7 and 5.8 have duals for $\hat{M}$ which we have not discussed. However, the dual lemmas can be proved just as in [5] since we defined the lists $\mathscr{C}_{s}^{\prime}, \mathscr{C l}_{s}^{\prime}, \mathscr{P}_{s}^{\prime}$ just as in [5] because we have no new rules removing elements of $\hat{M}$ to hole $\hat{H}_{4}$.

The next lemma establishes that Rule $R_{3}$ does not require too much enumeration. Let $\mathscr{S}^{e}(X)=\left\{\nu: \nu \in \mathscr{S}^{e}(X)\right.$ and $\left.|\nu| \leqq e\right\}$.

Lemma 5.9. Fix e. If each $\nu$ such that $|\nu|<e$ is added to $\mathscr{C}$ at most finitely often then $\hat{C}$ dual covers $\mathscr{S}\left(C_{3}\right)$.

Proof. Soare gives a proof [5, Lemma 5.4, p. 108] which depends only on Lemma 5.2 and Rule $R_{3}$.

Nevertheless, Rule $R_{3}$ allows enough enumeration to prove the following key lemma.

Lemma 5.10. If $\hat{X}$ is any stream of $\hat{M}$, and $C$ covers $\hat{X}$, then $D$ r-exactly covers $\hat{X}$.

Proof. (This is essentially the same proof as that of [5, Lemma 5.6, p. 109] which has similar content.)

Fix a stream $\hat{X}$ of $\hat{M}$, and assume for a contradiction that some $\nu_{1} \in \mathscr{S}(\hat{X})$ i.o. and $C$ covers $\nu_{1}$ but no $\nu \in \mathscr{S}(D)$ i.o., if $\nu_{1} \leqq^{\tau} \nu$. Let $\nu_{1}=\left\langle e, \sigma_{1}, \tau_{1}\right\rangle$. Then there exists $s^{\prime}$ such that no $\nu$ is added to $\mathscr{S}(D)$ at any stage $s \geqq s^{\prime}$ if $\nu_{1} \leqq^{\tau}[\nu]_{e}$. Choose $e^{\prime} \geqq e$ and $e^{\prime}>|\nu|$ for any $\nu \in \mathscr{S}(D)$ such that $\nu_{1} \leqq^{\tau}[\nu]_{e}$. Replacing $\nu_{1}$ by some extension if necessary we may assume that $\nu_{1} \in \mathscr{S}(\hat{X})$ i.o., $C$ covers $\nu_{1}$, and

$$
(\forall \nu)\left[\nu_{1} \leqq[\nu]_{e} \Longrightarrow \nu \notin \mathscr{S}(D)\right] \text {. }
$$


$\nu_{1} \notin \mathscr{P}_{s}$ for any $s$. Thus, by the definition of $\mathscr{H}$, each $\nu \in \mathscr{H}$ i.o. if $\nu_{1} \leqq^{\tau} \nu$.

Since $C$ covers $\nu_{1}$, some $\nu_{2}=\left\langle e, \sigma_{2}, \tau_{0}\right\rangle \in \mathscr{S}(\mathrm{C})$ i.o. (and hence $\nu_{2} \in \mathscr{S}\left(C_{1}\right)$ i.o.) where $\sigma_{2} \supseteqq \sigma_{1}$ and $\tau_{0} \supseteqq \tau_{1}$. Furthermore, $\nu_{1} \in \mathscr{S}_{8}(\hat{X})-$ $\mathscr{P}_{s}$ for infinitely many $s$ implies that $\nu_{3}=\left\langle e, \sigma_{2}, \tau_{1}\right\rangle \in \mathscr{H}$ i.o.

Now $\nu_{1} \leqq^{\tau} \nu_{3}$, and thus by $(6) \nu_{3} \notin \mathscr{S}(D)$. Hence, $\nu_{3} \notin \mathscr{S}\left(C_{3}\right)$ and $\nu_{3}$ once added to $\mathscr{C}$ is never checked under Rule $R_{3}$. Choose $s_{0}$ such that no $\nu$ preceding $\nu_{3}$ on the sequence $\mathscr{H}$ is checked at any stage $s \geqq s_{0}$. Choose $s_{1} \geqq s_{0}$ such that some $x \geqq e$ enters track $C_{1}$ at stage $s_{1}$, where $\nu\left(s_{1}, e, x\right)=\nu_{2}=\left\langle e, \sigma_{2}, \tau_{0}\right\rangle$. But $\tau_{0} \subseteq \tau_{1}$, and hence at stage $s+1$ by Rule $R_{3}, \nu_{3}=\left\langle e, \sigma_{2}, \tau_{1}\right\rangle$ is checked, and $x$ is placed on track $C_{3}$ with $\nu\left(s_{1}+1, e, x\right)=\nu_{3}$, contrary to (6).

LEMma 5.11. For each track $X$ of $M(\hat{X}$ or $\hat{M})$ and each $\nu$,

(1) $\nu \in \mathscr{S}(\hat{X})$ i.o. $=\nu \in \mathscr{P}_{\omega}$, and

(2) $\nu \in \mathscr{S}(X)$ i.o. $=\nu \in \mathscr{P}_{\omega}^{\prime}$.

The proof is by induction on the length of $\nu$. Fix $e$ and suppose that (1) and (2) hold for all $\nu$ with $|\nu|<e$. It suffices to prove (1) for all $\nu$ of length $e$ since (2) is dual. By inductive hypothesis (2), each $\nu$ of length $<e$ is added to $\mathscr{H}^{\prime}$ only finitely often. Thus by the dual of Lemma $5.9, C$ covers $\mathscr{S}^{e}\left(\widehat{C}_{3}\right)$.

Now inductive hypothesis (1), each $\nu$ of length $e$ is excluded from $\mathscr{M}_{s+1}$ under Condition 2 of Definition 4.7(a) only finitely often. Thus for every $\nu$ of length $e$ we have

$$
\begin{aligned}
& \nu \in \mathscr{S}(D) \text { i.o. and } \nu \in \mathscr{K}_{\omega} \Longrightarrow \nu \in \mathscr{K}_{\omega} \\
& \nu \in \mathscr{P}_{s} \text { for infinitely many } s \Longrightarrow \nu \in \mathscr{P}_{\omega} .
\end{aligned}
$$

Now assume for a contradiction that for some $\hat{X}$ and $\nu_{1}, \nu_{1} \epsilon$ $\mathscr{S}(\hat{X})$ i.o., but $\nu_{1} \notin \mathscr{P}_{\omega}$. Let $\nu_{1}=\left\langle e, \sigma_{1}, \tau_{1}\right\rangle$ with $\sigma_{1}$ minimal for $e$, and $\tau_{1}$ minimal for $e$ and $\sigma_{1}$. Since $\nu_{1} \notin \mathscr{P}_{\omega}, \nu_{1} \in \mathscr{P}_{s}$ for finitely many $s$, and thus

$$
\nu_{1} \in \mathscr{S}_{s}(\hat{X})-\mathscr{P}_{s} \text { for infinitely many } s .
$$

But for each such $s$ all $\nu^{\prime}$ of length $>e$ are excluded from $\mathscr{K}_{s+1}$ under Condition 2 and hence

$$
\nu \in \mathscr{C}_{\omega} \Longrightarrow|\nu| \leqq e .
$$

Now choose elements $\hat{y}_{j}$ and corresponding stages $s_{j}+1$ such that for all $j \in N$,

$$
\nu_{1}=\nu\left(s_{j}+1, e, \widehat{y}_{j}\right) \neq \nu\left(s_{j}, e, \widehat{y}_{j}\right) .
$$

Note that $s_{j}$ exists since $\nu\left(v_{j}, e, \hat{y}_{j}\right)=\langle e, \phi, \phi\rangle \in \mathscr{P}_{\omega}$ where $v_{j}$ is the 
stage that $\hat{y}_{j}$ enters $\hat{M}$. For each $j \in N$, define the finite sequence of full $e$-states,

$$
\mathscr{F}_{j}=\left\{\nu:(\exists s)\left[v_{j} \leqq s \leqq s_{j}+1 \text { and } \nu=\nu\left(s, e, \widehat{y}_{j}\right)\right]\right\},
$$

where $v_{j}$ is the stage when $\hat{y}_{j}$ entered $\hat{M}$. Let $\mathscr{F}$ be the concatenation of $\left\{\mathscr{F}_{j}: j \in N\right\}$. From the definition of $\mathscr{F}$, note that if $\nu \in \mathscr{F}$ i.o., then $\nu^{\prime} \in \mathscr{H}$ i.o. for all $\nu^{\prime}$ such that $\nu \leqq{ }^{\tau} \nu^{\prime}$. Thus by Rule $R_{3}$ we have

$$
\nu \in \mathscr{F} \text { i.o. and } C \text { covers } \nu \Longrightarrow D \tau \text {-exactly covers } \nu \text {. }
$$

But $\nu_{1} \notin \mathscr{P}_{\omega}$, so $D$ does not $\tau$-exactly cover $\nu_{1}$ and hence

$$
C \text { does not cover } \nu_{1} \text {. }
$$

On the other hand we shall get a contradiction from this by proving that

$$
C \text { covers } \mathscr{F} \text {. }
$$

First note that by the minimality of $\sigma_{1}$ and $\tau_{1}$ above we have,

$$
\nu \in \mathscr{F} \text { i.o. and } \nu \neq \nu_{1} \Longrightarrow \nu \in \mathscr{P}_{\omega},
$$

and thus,

$$
\text { (a.a.j) }(\forall s)_{\geqq_{j}}\left[d\left(s, y_{j}\right) \geqq e\right] \text {, }
$$

by the definition of $d(s, y)$ from $\mathscr{P}_{s}$, and by induction on $t$ for $v_{j} \leqq t \leqq s_{j}$.

Assume for a contradiction that $C$ does not cover $\mathscr{F}$, and choose $\nu_{0}=\left\langle e, \sigma_{0}, \tau_{0}\right\rangle$ such that

$$
\nu_{0} \in \mathscr{F} \text { i.o. but } C \text { does not cover } \nu_{0},
$$

where $\sigma_{0}$ and $\tau_{0}$ are minimal. Choose an infinite set $J$ and stages $t_{j}, t_{j}+1 \leqq s_{j}+1$ such that

$$
(\forall j \in J)\left[\nu_{0}=\nu\left(t_{j}+1, e, \hat{y}_{j}\right) \neq \nu\left(t_{j}, e, \widehat{y}_{j}\right)\right] .
$$

(Of course, $t_{j}+1>v_{j}$ since $\hat{y}_{j}$ changes $e$-state only when in machine $\hat{M}$.)

$$
C \text { covers the sequence }\left\{\nu\left(t_{j}, e, \widehat{y}_{j}\right): j \in J\right\} \text {. }
$$

Now by Rule $\hat{R}_{6}$, for almost all $j \in J$, either Rule $\hat{R}_{3}$ or $\hat{R}_{4}$ applies to $\hat{y}_{j}$ at stage $t_{j}+1$. Now Rule $\hat{R}_{3}$ applies for at most finitely many $j \in J$ because if Rule $\hat{R}_{3}$ applies infinitely often, $\nu_{0} \in \mathscr{S}\left(\hat{C}_{3}\right)$ i.o. but then $C$, which covers $\mathscr{S}^{e}\left(\widehat{C}_{3}\right)$, would cover $\nu_{0}$.

Thus, for almost all $j \in J$, Rule $\hat{R}_{4}$ applies to $\hat{y}_{j}$ at stage $t_{j}+1$, 
with $\nu_{0} \precsim \nu^{\prime \prime}\left(t_{j}+1\right)$. However, $\mathscr{K}_{s}\left[\nu_{0}\right] \subseteq\left\{\nu_{0}\right\}$ for almost all $s$ since $\nu \in \mathscr{M}_{\omega} \Rightarrow|\nu| \leqq e$ and $D$ does not $\tau$-exactly cover $\nu_{0}$. Hence, $\nu^{\prime \prime}\left(t_{j}+1\right)=$ $\nu_{0}$ for almost all $j \in J$. Fix any $\nu_{0}^{\prime}$ such that $\nu_{0}^{\prime}=\nu^{\prime}\left(t_{j}+1\right)$ in Rule $\hat{R}_{4}$ for infinitely many $j \in J$. $C$ covers $\nu_{0}^{\prime}$, and $\nu_{0}^{\prime} \in \mathscr{F}$ i.o., so $D \tau$ exactly covers $\nu_{0}^{\prime}$. Thus, by Lemma 5.8,D $\tau$-exactly covers $\nu_{0}$, and therefore $C$ covers $\nu_{0}$ which is the desired contradiction.

Lemma 5.12. $C$ covers $\hat{C}_{3}$ and $\hat{C}$ dual covers $C_{3}$.

Proof. The proof in [5, Lemma 5.12, p. 113] depends only on Lemma 5.11 and Rule $R_{3}\left(\hat{R}_{3}\right)$.

LEMMA 5.13. ( $\forall e)($ a.a.s) (a.a. $\hat{y})\left[\hat{y} \in \widehat{M}_{s}-\hat{A}_{s} \Rightarrow d(s, \hat{y}) \geqq e\right]$.

Proof. [5, Lemma 5.13, p. 113]. (This uses only Lemma 5.11 and the definition of $d$.)

Lemma 5.14. Given $\nu_{1}$ and infinitely many elements $\hat{y}_{j}, j \in N$, such that for all $j \in N$, Rule $\hat{R}_{4}$ applies to $\hat{y}_{j}$ at stage $s_{j}$ with $\nu_{1} \precsim$ $\nu\left(s_{j}+1, y_{j}, \widehat{y}_{j}\right)$, then $C$ covers $\nu_{1}$.

Proof. The proof [5, Lemma 5.14, p. 113] depends only on Lemma 5.13 and Rule $\hat{R}_{4}$.

Lemma 5.15. C covers every stream $\hat{X}$ of $\hat{M}, \hat{X} \neq \hat{A}$.

Proof. [5, Lemma 5.15, p. 113]. The proof depends on Lemma 5.12 and Lemma 5.14.

LEMMA 5.16. $D$-exactly covers every stream $\hat{X}$ of $\hat{M}, \hat{X} \neq \hat{A}$.

Proof. Lemmas 5.15 and 5.10.

Of course the dual Lemmas 5.13-5.16 have similar proofs.

The next lemma is crucial for constructing the permutation of $\bar{A}$ to $\bar{B}$. The statement of the lemma is the same as [5, Lemma 6.1] but the proof is different due to the complications of our construction. It is here that the condition 3 exclusion of Definition 4.7(a) plays a crucial role. If $\hat{q}\left(\nu_{0}\right)=\lim _{s} \hat{q}\left(s, \nu_{c}\right)$ exists but $p\left(\nu_{0}\right)$ does not, we would like it to be the case that $\hat{q}(\nu)$ exists for only finitely many $\nu \succsim \nu_{0}$. This is done by arranging that $\mathscr{M}_{\omega}\left[\nu_{0}\right]$ is finite since if $\hat{x} \in \hat{Q}_{\omega}, \nu^{*}(\hat{x}) \in \mathscr{M}_{\omega}$. As in [5], condition 1 exclusion handles the cases where $p\left(s, \nu_{0}\right)$ becomes undefined due to changes in $\left|\nu_{0}\right|$-state. 
Condition 3 was designed to handle the case in which $p\left(s, \nu_{0}\right)$ becomes undefined because $p\left(s, \nu_{0}\right)$ enters $A$.

LEMMA 5.17. Fix $\nu_{0}$ and suppose that

(1) $\left(\forall \nu \prec \nu_{0}\right)\left[\lim _{s} p(s, \nu)\right.$ exists],

(2) $\left(\forall \nu \precsim \nu_{0}\right)\left[\lim _{s} \widehat{q}(s, \nu)\right.$ exists], and

(3) $\lim _{s} p\left(s, \nu_{0}\right)$ does not exist.

Then $\mathscr{H}_{\omega}\left[\nu_{0}\right]$ is finite.

Proof. Suppose that $\nu_{0}=\left\langle e_{0}, \sigma_{0}, \tau_{0}\right\rangle$ and let $s_{0}$ be a stage such that

$$
\begin{aligned}
& \left(\forall \nu \prec \nu_{0}\right)\left[p\left(s_{0}, \nu\right)=p(\nu)\right], \quad \text { and } \\
& \left(\forall \nu \precsim \nu_{0}\right)\left[\hat{q}\left(s_{0}, \nu\right)=\hat{q}(\nu)\right] .
\end{aligned}
$$

We will show that, if $\nu_{1} \in \mathscr{C}_{s_{1}}\left[\nu_{0}\right]-\mathscr{C}_{s_{1}-1}\left[\nu_{0}\right]$ for some $s_{1}>s_{0}$ and $r\left(s_{1}, \nu_{1}\right)>s_{0}$, then $\nu_{1}$ is excluded from $\mathscr{M}_{t+1}$ for some stage $t \geqq s_{1}$. (This clearly implies that $\mathscr{L}_{\omega}\left[\nu_{0}\right]$ is finite since $\mathscr{L}_{\omega}\left[\nu_{0}\right] \subseteq$ $\mathscr{C}_{s_{0}}\left[\nu_{0}\right] \cup\left\{\nu_{k}: k \leqq s_{0}\right\}$.) If $\nu_{1}$ is never excluded from $\mathscr{M}_{t+1}$ for $t \geqq s_{1}$, by Condition 1 of Definition 4.7(a), it must be the case that $\lim _{s} r(s, \nu)=r\left(s_{1}, \nu\right)$ for all $\nu$ such that $r\left(s_{1}, \nu\right) \leqq r\left(s_{1}, \nu_{1}\right)$.

Since $\nu_{1} \in \mathscr{C}_{s_{1}}-\mathscr{H}_{s_{1}-1}$, some element $x$ is on track $D$ at stage $s_{1}$ in state $\nu\left(s_{1}, x, x\right)$ such that

$$
\nu_{0} \lesssim \nu_{1} \lesssim \nu\left(s_{1}, x, x\right) .
$$

By Rule $R_{2}, x$ is preferred as $p\left(s_{1}+1, \nu_{0}\right)$ unless $\rho\left(s_{1}+1, \nu_{0}, p\left(s_{1}, \nu_{0}\right)\right) \leqq$ $r\left(s_{1}+1, \nu_{1}\right)$. Thus, in any case,

$$
\rho\left(s_{1}+1, \nu_{0}, p\left(s_{1}+1, \nu_{0}\right)\right) \leqq r\left(s_{1}+1, \nu_{1}\right) .
$$

Now $p\left(s, \nu_{0}\right)=p\left(s+1, \nu_{0}\right)$ unless Rule $R_{2}, R_{10}, R_{11}, R_{D}$ or Subcase $3(\mathrm{c})$ of the construction removes it. It is easy to see that Rules $R_{11}$ and $R_{D}$ never apply to $p\left(s, \nu_{0}\right)$ for any $s>s_{0}$ since $p\left(s_{0}, \nu^{\prime}\right)=p\left(\nu^{\prime}\right)$ for every $\nu^{\prime} \prec \nu_{0}$. Rule $R_{2}$ can remove $p\left(s, \nu_{0}\right)$ from $P_{s}$ in two ways after stage $s_{0}$ : by placing $p\left(s, \nu_{0}\right)$ above hole $H_{4}$ at stage $s+1$ or by defining $p\left(s+1, \nu_{0}\right)$ to be a new element. Suppose Rule $R_{2}$ is used to replace $p\left(s, \nu_{0}\right)$ at some state $s_{2}+1>s_{1}+1$ before any of the other Rules are used to remove $p\left(s, \nu_{0}\right)$. Then, by induction on stages $s$ such that $s_{1}+1 \leqq s \leqq s_{2}$,

$$
\rho\left(s_{2}, \nu_{0}, p\left(s_{2}, \nu_{0}\right)\right)=\rho\left(s_{1}+1, \nu_{0}, p\left(s_{1}, \nu_{0}\right)\right) \leqq r\left(s_{1}+1, \nu_{1}\right) .
$$

This is true by Lemma 4.3 , the fact that $p\left(s_{2}, \nu_{0}\right)=p\left(s_{1}, \nu_{0}\right)$ does not change in state while in pocket $P$, and the fact that $\lim _{s} r(s, \nu)=$ $r\left(s_{1}, \nu\right)$ for all $\nu$ such that $r\left(s_{1}, \nu\right) \leqq r\left(s_{1}, \nu_{1}\right)$. At stage $s_{2}+1, \rho\left(s_{2}, \nu_{0}\right.$, $\left.p\left(s_{2}+1, \nu_{n}\right)\right)<\rho\left(s_{2}, \nu_{0}, p\left(s_{2}, \nu_{0}\right)\right)$. Thus, by induction on stages $s>s_{1}$, 
$\rho\left(s, \nu_{0}, p\left(s, \nu_{0}\right)\right)$ decreases with each application of Rule $R_{2}$ to $p\left(s, \nu_{0}\right)$ as long as no other rule intervenes.

Thus we have shown that there must be a stage $s+1>s_{1}+1$ such that $p\left(s, \nu_{0}\right)$ is removed from pocket $P$ at stage $s+1$ by being removed to hole $H_{4}$ (by Subcase $3\left(\mathrm{c}\right.$ ) or Rule $R_{2}$ ) or by Rule $R_{10}$. Furthermore, $\rho\left(s, \nu_{0}, p\left(s, \nu_{0}\right)\right) \leqq r\left(s_{1}+1, \nu_{1}\right)$.

If $p\left(s, \nu_{0}\right)$ is removed to hole $H_{4}$, it is easy to see that $\nu_{1}$ is excluded from $\mathscr{M}_{s+1}$ by condition 3 of Definition 4.7(a) since

$$
r\left(s_{1}, \nu_{1}\right)>s_{0} \geqq(\mu t \leqq s)(\forall z)_{t \leqq z \leqq s}\left(\forall \nu^{\prime} \lesssim \nu_{0}\right)\left[\hat{q}\left(z, \nu^{\prime}\right)=\hat{q}\left(s, \nu^{\prime}\right)\right]
$$

and

$$
r\left(s_{1}, \nu_{1}\right) \geqq \rho\left(s, \nu_{0}, p\left(s, \nu_{0}\right)\right) \text {. }
$$

Suppose, on the other hand, that $p\left(s, \nu_{0}\right)$ is removed from pocket $P$ at stage $s+1$ by Rule $R_{10}$. Then

$$
\nu\left(s+1, e_{0}, p\left(s, \nu_{0}\right)\right)^{\tau}>\nu\left(s, e_{0}, p\left(s, \nu_{0}\right)\right)
$$

and, in fact, if the state corresponding to $\rho\left(s, \nu_{0}, x\right)$ is $\left\langle e_{1}, \sigma_{1}, \tau_{1}\right\rangle$, $\nu\left(s+1, e_{1}, p\left(s, \nu_{0}\right)\right){ }^{\tau}>\nu\left(s, e_{1}, p\left(s, \nu_{0}\right)\right)$ since $e_{0} \leqq e_{1}$. But then at some later stage $t+1>s+1, p\left(s, \nu_{0}\right)$ enters track $C$ in full $e_{1}$-state $\nu\left(s+1, e_{1}, p\left(s, \nu_{0}\right)\right)$ by Lemma 5.4. This causes $\nu_{1}$ to be excluded from $\mathscr{C}_{s+1}$ by condition 1 and the fact that $r\left(s_{1}+1, \nu\right) \geqq \rho\left(s, \nu_{0}\right.$, $\left.p\left(s, \nu_{0}\right)\right)$ and $\nu\left(s, e_{1}, p\left(s, \nu_{0}\right)\right) \in \mathscr{K}_{t+1}^{2}$. This last contradiction shows that $\nu_{1}$ must in fact be excluded from $\mathscr{K}_{s}$, for some $s>s_{1}$.

LEMMA 5.18. Fix $\nu$ and suppose that there are infinitely many $\hat{y}_{j} \in \widehat{Q}_{\omega}, j \in N$, such that $\nu \precsim \nu\left(y_{j}, \hat{y}_{j}\right), j \in N$. Then $\lim _{s} p(s, \nu)$ exists.

Proof. By the definition of $\nu^{*}$ and Lemma 5.13 it is clear that $\nu \precsim \nu^{*}\left(\hat{y}_{j}\right)$ for almost every $j$. Now by Lemma 5.14 , if $\lim _{s} p(s, \nu)$ does not exist, then $\mathscr{L}_{\omega}[\nu]$ is finite. But if $\hat{y}_{j} \in \hat{Q}_{\omega}, \nu^{*}\left(\hat{y}_{j}\right) \in \mathscr{H}_{\omega}$ by Lemma 4.8. For any particular $\nu_{0}, \nu^{*}\left(\widehat{y}_{j}\right)=\nu_{0}$ for only finitely many $j$. This is a contradiction.

Recall that the desired permutation of $\bar{A}$ to $\bar{B}$ was the one that sent $\hat{q}(v)$ to $p(\nu)$ and $q(\nu)$ to $\hat{p}(\nu)$ for every full $e$-state $\nu$. Of course, this is not quite right since $\hat{q}(\nu)$ may exist where $p(\nu)$ does not. In this case, however, Lemma 5.18 suggests a finite-one map which will suffice.

LEMMA 5.19. There are finite-one maps $p_{1}: \hat{Q}_{\omega} \rightarrow P_{\omega}$ and $p_{2}: Q_{\omega} \rightarrow$ $\hat{P}_{\omega}$ such that for every $n$
(1)
$p_{1}\left(\hat{Q}_{\omega} \cap\right.$
$\left.\hat{U}_{n}^{+}\right)={ }^{*} P_{\omega} \cap U_{n}$ 
(2) $p_{1}\left(\hat{Q}_{\omega} \cap V_{n}\right)={ }^{*} P_{\omega} \cap \hat{V}_{n}^{+}$,

(3) $p_{2}\left(Q_{\omega} \cap \hat{V}_{n}^{+}\right)={ }^{*} \hat{P}_{\omega} \cap V_{n}$, and

(4) $p_{2}\left(Q_{\omega} \cap U_{n}\right)={ }^{*} \hat{P}_{\omega} \cap \hat{U}_{n}^{+}$.

Proof. We define $p_{1} ; p_{2}$ is similar. If $p(\nu)$ is defined, let $p_{1}(q(\nu))=$ $p(\nu)$. If $p(\nu)$ is undefined, let $\nu^{\prime} \lesssim \nu$ the maximal full $e$-state in the ordering $\lesssim$ such that $p\left(\nu^{\prime}\right)$ is defined and define $p_{1}(q(\nu))=p\left(\nu^{\prime}\right)$. This map is finite-one by Lemma 5.18 and meets (1) and (2) since $q(\nu)$ and $p(\nu)$ are in the same full $|\nu|$-state.

Although we have not given a one-to-one map from $\bar{A}$ to $\bar{B}$ inducing an automorphism on $\bar{A}$, the details on constructing such a permutation are straightforward, given Lemma 5.19, and can be found in [5, Corollary 1.7, p. 85]. Notice that $\bar{A}$ is infinite, since $\bar{B}$ is. Also, note that $U_{n}={ }^{*} V_{n}={ }^{*} W_{n}$, since if $x \in W_{n}, x \in U_{n}\left(x \in V_{n}\right)$ unless $x=p(\nu)(\hat{x}=\hat{p}(\nu))$ for some $\nu$ such that $|\nu|<n$. (Note that $x$ cannot be in pocket $P$ infinitely often without being $p(\nu)$ for some $\nu$ since $x$ enters $P$ only finitely often by Lemma 5.1.)

We can now show that the hypothesis of the Extension Theorem [5, Theorem 2.2] are met by our enumeration of $A$ and $B$. It is easy to see that this hypothesis is simply

LEMMA 5.20. $C_{\vartheta}$ covers $\hat{C}_{9} ; \hat{C}_{9}$ dual covers $C_{9}$.

Proof. $\hat{C}$ dual covers every stream $X$ of $M$. Thus $\hat{C}_{6}$ dual covers every stream $X$ of $M$ by Lemma 4.1. Suppose $\nu \in \mathscr{S}\left(C_{\vartheta}\right)$ i.o. Then there are infinitely many $\nu^{\prime} \in \mathscr{S}\left(\widehat{C}_{6}\right)$ such that $\nu^{\prime} \leqq \nu$. Suppose $\nu$ appears as the eth element of $\mathscr{S}_{\unlhd_{s}}\left(C_{\theta}\right)$. Then at some stage $s_{0}>s$ some $x$ not in $\bigcup_{i \leqq e} D_{h(i)}$ must enter $\widehat{C}_{6}$ in full $e$-state $\nu^{\prime} \leqq \nu$. Thus, $x$ checks $\nu$ and enters $\hat{C}_{8}$ by Rule $\hat{R}_{B}$. Thus $\hat{C}_{9}$ dual covers $C_{9}$ since any $x$ checks only finitely many occurrences of $\nu$ on $\mathscr{S}_{s}\left(C_{\vartheta}\right)$ and each occurrence is eventually checked.

The proof that $C_{9}$ covers $\hat{C}_{9}$ is similar. Instead of using some $x$ not in $\bigcup_{i \leqq e} D_{h(i)}$ to cover the eth element on $\mathscr{S}\left(\hat{C}_{9}\right)$, we use any $x$. Since $C_{6}$ covers $\hat{C}_{9}$, eventually some such $x$ in the appropriate $e$-state appears on track $C_{6}$.

Lemma 5.21. A is dense simple.

Proof. Subcase 3(c) of Step 1 of the construction guarantees that $p_{\bar{A}}$ dominates every (partial) recursive function.

Lemma 5.21 shows that $A$ is dense simple, Lemma 5.3 that $B$ is not hypersimple. Lemma 5.20 and the Extension Theorem of 
Soare guarantee the existence of a permutation $p$ and r.e. sets $\left\{\hat{U}_{n}^{-}\right\}_{n \in N}$ and $\left\{\hat{V}_{n}^{-}\right\}_{n \in N}$ extending our permutation of Lemma 5.19 to an automorphism of $\mathscr{E}^{*}$ taking $A^{*}$ to $B^{*}$.

6. Open questions. If $B$ is any simple set, is there a dense simple set $A$ and an automorphism $\Phi$ of $\mathscr{E}$ such that $\Phi(A)=B$ ? It is known that $A$ can be found such that $\mathscr{L}^{*}(B) \cong \mathscr{L}^{*}(A)$.

What is the class of r.e. sets $A$ such that $\Phi(A)$ is hypersimple for every automorphism $\Phi$ of $\mathscr{E}$ ? Only trivial results about this question are known.

Is the property of strong hypersimplicity invariant? $(A$ is strongly hypersimple if there does not exist a recursive function $f$ such that $\bigcup_{n} W_{f(n)}=N, W_{f(n)} \cap \bar{A} \neq \phi$ for each $n$, and the r.e. sets $W_{f(n)}$ are finite and pairwise disjoint.) This question may require yet another modification of the automorphism machinery to answer.

Much recent research has identified new properties of r.e. sets which are invariant under Aut (E⿱E) (see [1], [2], and [4] for example). Can the methods now known for constructing automorphisms of $\mathscr{E}$

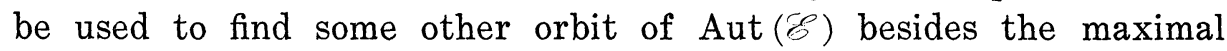
sets?

\section{REFERENCES}

1. M. Lerman and R. I. Soare, d-simple sets, small sats, and degree classes, to appear.

2. M. Lerman, R. A. Shore, and R. J. Soare, r-maximal major subsets, Israel J. Math., 31 (1978), 1-18.

3. H. Rogers, Jr., Theory of Recursive Functions and Effective Computability, McGraw-Hill, N.Y., 1967.

4. R. A. Shore, Determining automorphism of recursively enumerable sets, to appear.

5. R. I. Soare, Automorphisms on the lattice of recursively enumerable sets, Part I: Maximal sets, Annals Math., 100 (1974), 80-120.

6. - Automorphisms of the lattice of recursively enumerable sets. Part II: Low sets, to appear.

Received April 18, 1980. This work first appeared as part of the author's doctoral dissertation. The author wishes to thank his advisor, Prof. Robert I. Soare, for the information, comments, and encouragement he provided during the preparation of the thesis and this paper. The author was partially supported by National Science Foundation Grants MCS-76-07033 and MCS-80-02937.

MASSAChUSETTS Institute of TeChNOLOGY

CAMBRidge, MA 02139

Current address: Calvin College Grand Rapids, MI 49506 



\section{PACIFIC JOURNAL OF MATHEMATICS}

\section{EDITORS}

DONALD BABBITT (Managing Editor)

University of California

Los Angeles, CA 90024

Hugo RossI

University of Utah

Salt Lake City, UT 84112

C. C. Moore and Arthur Agus

University of California

Berkeley, CA 94720
J. DugundJI

Department of Mathematics

University of Southern California

Los Angeles, CA 90007

R. FinN and J. MiLgRAM

Stanford University

Stanford, CA 94305

\section{ASSOCIATE EDITORS}
R. ARENS
E. F. BECKENBACH
B. H. NeumanN
F. WOLF
K. YoSHIDA

\section{SUPPORTING INSTITUTIONS}

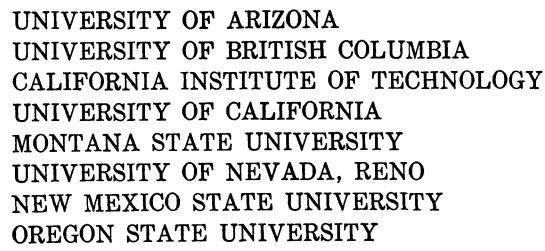

UNIVERSITY OF ARIZONA

UNIVERSITY OF BRITISH COLUMBIA CALIFORNIA INSTITUTE OF TECHNOLOGY UNIVERSITY OF CALIFORNIA MONTANA STATE UNIVERSITY

UNIVERSITY OF NEVADA, RENO NEW MEXICO STATE UNIVERSITY OREGON STATE UNIVERSITY

\author{
UNIVERSITY OF OREGON \\ UNIVERSITY OF SOUTHERN CALIFORNIA \\ STANFORD UNIVERSITY \\ UNIVERSITY OF AAWAII \\ UNIVERSITY OF TOKYO \\ UNIVERSITY OF UTAH \\ WASHINGTON STATE UNIVERSITY \\ UNIVERSITY OF WASHINGTON
}

The Supporting Institutions listed above contribute to the cost of publication of this Journal, but they are not owners or publishers and have no responsibility for its content or policies,

Mathematical parers intended for publication in the Pacific Journal of Mathematics should be in typed form or offset-reproduced, (not dittoed), double spaced with large margins. Please do not use built up fractions in the text of the manuscript. However, you may use them in the displayed equations. Underline Greek letters in red, German in green, and script in blue. The first paragraph or two must be capable of being used separately as a synopsis of the entire paper. Please propose a heading for the odd unmbered pages of less than 35 characters. Manuscripts, in triplicate, may be sent to any one of the editors. Please classify according to the scheme of Math. Reviews, Index to Vol. 39. Supply name and address of author to whom proofs should be sent. All other communications should be addressed to the managing editor, or Elaine Barth, University of California, Los Angeles, California, 90024 .

50 reprints to each author are provided free for each article, only if page charges have been substantially paid. Additional copies may be obtained at cost in multiples of 50 .

The Pacific Journal of Mathematics is issued monthly as of January 1966, Regular subscription rate: $\$ 114.00$ a year (6 Vol., 12 issues). Special rate: $\$ 57.00$ a year to individual members of supporting institution.

Subscriptions, orders for numbers issued in the last three calendar years, and changes of address shoud be sent to Pacific Journal of Mathematics, P.O. Box 969, Carmel Valley, CA 93924, U.S.A. Old back numbers obtainable from Kraus Periodicals Co., Route 100, Millwood, NY 10546.

\section{PUBLISHED BY PACIFIC JOURNAL OF MATHEMATICS, A NON-PROFIT CORPORATION}

Printed at Kokusai Bunken Insatsusha (International Academic Printing Co., Ltd.). 8-8, 3-chome, Takadanobaba, Shinjuku-ku, Tokyo 160, Japan.

Copyright (C) 1982 by Pacific Journal of Mathematics Manufactured and first issued in Japan 


\section{Pacific Journal of Mathematics}

Vol. 100, No. $2 \quad$ October, 1982

Kenneth F. Andersen, On the transformation of Fourier coefficients of

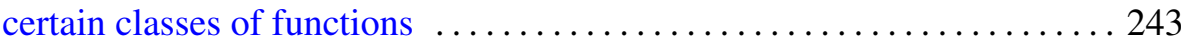

Steven Albert Bleiler, Realizing concordant polynomials with prime knots

Reinhard Bürger, Functions of translation type and solid Banach spaces of functions

Ulrich Daepp, The saturation of $k$-analytic rings and topological equivalence of associated analytic set germs .................. 271

Persi W. Diaconis and David Amiel Freedman, On the maximum difference between the empirical and expected histograms for sums . . . 287

David Amiel Freedman, On the maximum of scaled multinomial

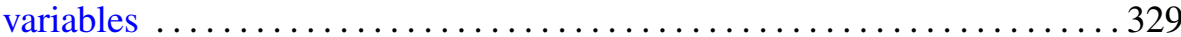

Persi W. Diaconis and David Amiel Freedman, On the difference between the empirical histogram and the normal curve, for sums. II ......... 359

Persi W. Diaconis and David Amiel Freedman, On the mode of an

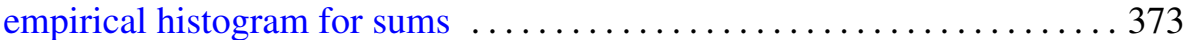

Jutta Hausen, Supplemented modules over Dedekind domains 387

Elyahu Katz, A moduli representation for the classification of twisted tensor

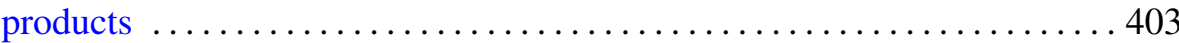

H. C. Madhekar and N. K. Thakare, Biorthogonal polynomials suggested by the Jacobi polynomials

Ted R. Pettis, Collections of covers of metric spaces 425

Ryōtarō Satō, Maximal functions for a semiflow in an infinite measure space

Michael Jay Stob, Invariance of properties under automorphisms of the lattice of recursively enumerable sets 